\title{
Spatially Aware Dimension Reduction for Spatial Transcriptomics
}

\author{
Lulu Shang ${ }^{1,2}$, Xiang Zhou ${ }^{1,2}$
}

4 1. Department of Biostatistics, University of Michigan, Ann Arbor, MI 48109, USA

2. Center for Statistical Genetics, University of Michigan, Ann Arbor, MI 48109, USA

\#: correspondence to XZ (xzhousph@umich.edu)

\section{Abstract}

Spatial transcriptomics are a collection of genomic technologies that have enabled transcriptomic profiling on tissues with spatial localization information. Analyzing spatial transcriptomic data is computationally challenging, as the data collected from various spatial transcriptomic technologies are often noisy and display substantial spatial correlation across tissue locations. Here, we develop a spatially-aware dimension reduction method, SpatialPCA, that can extract a low dimensional representation of the spatial transcriptomics data with enriched biological signal and preserved spatial correlation structure, thus unlocking many existing computational tools previously developed in single-cell RNAseq studies for tailored and novel analysis of spatial transcriptomics. We illustrate the benefits of SpatialPCA for spatial domain detection and explores its utility for trajectory inference on the tissue and for high-resolution spatial map construction. In the real data applications, SpatialPCA identifies key molecular and immunological signatures in a newly detected tumor surrounding microenvironment, including a tertiary lymphoid structure that shapes the gradual transcriptomic transition during tumorigenesis and metastasis. In addition, SpatialPCA detects the past neuronal developmental history that underlies the current transcriptomic landscape across tissue locations in the cortex.

Keywords: Dimension reduction, SpatialPCA, spatially resolved transcriptomic studies, spatial map. 


\section{Introduction}

Spatial transcriptomics is a collection of groundbreaking new genomics technologies that enable the measurement of gene expression with spatial localization information on tissues. Specifically, the next-generation DNA sequencing-based technologies represented by Slide-seq ${ }^{1 ;} 2$, hybridization based technologies represented by STARmap ${ }^{3}$, and spatial transcriptomics through spatial barcoding from $10 \mathrm{x}$ genomics ${ }^{4 ; 5}$, can measure tens of thousands of genes on thousands of tissue locations, each of which consisting of a few to a few dozen single cells. In situ RNA sequencing-based technologies, such as targeted in situ sequencing (ISS) ${ }^{6}$ and FISSEQ $^{7}$, can measure the entire transcriptome at the single-cell resolution. The single-molecular fluorescence in situ hybridization (smFISH)-based technologies, represented by MERFISH ${ }^{8-10}$, seqFISH ${ }^{11 ; 12}$, and seqFISH plus ${ }^{13}$, can measure hundreds to tens of thousands of genes on subcellular organelles inside single cells across the tissue. These technologies altogether have enabled the study of the spatial transcriptomic landscape of tissues, catalyzing new discoveries in many areas of biology ${ }^{14}$; 15 .

Regardless of which technology one uses, the expression measurements obtained from spatial transcriptomics, just like any other genomic data types, are often noisy ${ }^{16}$. A common data processing step for extracting informative signals from noisy data in other genomic data types is dimension reduction. Dimension reduction aims to enrich biological signals through inferring a low-dimensional representation of the original genomic data. Dimension reduction is commonly applied to many genomics studies including the recent single cell RNA sequencing (scRNA-seq) studies ${ }^{17}$. In scRNA-seq, dimension reduction has become an indispensable data processing step for noise removal, facilitating data visualization and multiple downstream analyses that include cell type clustering ${ }^{18}$ and lineage inference ${ }^{19 ; 20}$. Many dimension reduction methods have been previously developed for scRNA-seq ${ }^{21-23}$ and some of these approaches have been directly applied to spatial transcriptomics. For example, Seurat ${ }^{24}$ recommends the use of principal component analysis (PCA) to preprocess spatial transcriptomics data. STUtility ${ }^{25}$ performs dimension reduction in spatial transcriptomics using non-negative matrix factorization (NMF), where the NMF factors are further interpreted through association analysis with distinct gene pathways. Despite these initial applications, however, scRNA-seq dimension reduction methods are not tailored for spatial transcriptomics and are not fully effective there. In particular, scRNA-seq based dimension reduction methods do not make use of the rich localization information contained in spatial transcriptomics and are not able to take advantage of the spatial correlation structure across tissue locations. Intuitively, in spatial transcriptomics, the neighboring locations on a tissue often share similar composition of cell types and display similar gene expression levels. Consequently, the low dimensional components on neighboring locations are likely to be similar to each other, more so than those on locations that are far away. Accounting for the similarity in the lowdimensional components on neighboring locations could facilitate effective dimension reduction in spatial transcriptomics and enable tailored downstream analyses.

In the present study, we explore the benefits of dimension reduction for a particular analytic task in spatial transcriptomics that is commonly referred to as spatial domain detection. Spatial domains represent spatially organized and functionally distinct anatomical structures on the tissue that are each characterized by unique local features with varying cell type composition, transcriptome heterogeneity, and cell-cell interactions ${ }^{26-28}$. Detecting spatial domains on the tissue is a critical 
first step towards understanding how these domains are coordinated with each other in carrying out the tissue functions and how they are generated through the complex developmental process.

80 Several methods have been recently developed for detecting spatial domains in spatial 81 transcriptomics, each with its own advantages and drawbacks. For example, BayesSpace ${ }^{29}$ detects spatial domains in spatial transcriptomics (ST) or 10x Visium data through explicit modeling of the specific spatial arrangement of the measured locations on the tissue. In the process of domain detection, BayesSpace can also enhance the spatial transcriptomics data with a fixed resolution, though it comes with a relatively heavy computational burden. As another example, $\mathrm{SpaGCN}^{30}$ detects spatial domains using a graph convolutional network, which relies on an adjacency matrix to incorporate spatial and histological information in the graph convolution layer. Despite its computational efficiency, as we will show here, SpaGCN is effective primarily in the setting where each spatial domain is dominated by one cell type. As a third example, stLearn ${ }^{31}$ extracts morphological features from an H\&E image that accompanies certain spatial transcriptomics technologies to perform spatial smoothing on the expression data, with which it performs spatial domain detection and further trajectory inference on pairs of spatial domains. However, the applications of stLearn are limited to spatial transcriptomics that collect H\&E images.

Here, motivated by the limitations of existing methods for dimension reduction and spatial domain detection in spatial transcriptomics, we develop a spatially aware dimension reduction method, which we refer to as the spatial probabilistic PCA, or SpatialPCA. SpatialPCA enables tailored dimension reduction in spatial transcriptomics and facilitates effective downstream analyses that include spatial domain detection. A unique feature of SpatialPCA is its ability to explicitly model the spatial correlation structure across tissue locations, thus preserving the neighboring similarity of the original data in the low dimensional manifold. The low dimensional components obtained from SpatialPCA contain valuable spatial correlation information and can be directly paired with existing computational tools developed in scRNA-seq for effective and novel downstream analysis in spatial transcriptomics. In particular, the low-dimensional components from SpatialPCA can be paired with scRNA-seq clustering methods to enable effective de novo tissue domain detection and can be paired with scRNA-seq trajectory inference methods to enable effective developmental 107 trajectory inference on the tissue. Because of the data generative nature of SpatialPCA and its 108 explicit modeling of spatial correlation, it can also be used to impute the low dimensional components on new and unmeasured arbitrary spatial locations, facilitating the construction of a refined spatial map with a resolution much higher than that measured in the original study. We illustrate the benefits of SpatialPCA through comprehensive simulations and applied it on four spatial transcriptomics datasets obtained with distinct technologies and tissue structures. 


\section{Results}

114

115

116

117

118

119

120

121

122

123

124

125

126

127

128

129

130

131

132

133

134

135

136

137

138

139

140

141

142

143

144

145

146

147

148

149

150

151

152

153

154

155

156

157

158

\section{Method overview}

SpatialPCA is described in the Methods section, with its technical details provided in Supplementary Text and its method schematic displayed in Figure 1a. Briefly, SpatialPCA is a spatially aware dimension reduction method that aims to infer a low dimensional representation of the gene expression data for spatial transcriptomics. SpatialPCA builds upon the probabilistic version of PCA, incorporates localization information as additional input, and uses a kernel matrix to explicitly model the spatial correlation structure across tissue locations. Because the inferred low dimensional components from SpatialPCA contain valuable spatial correlation information, we refer to these inferred components as spatial principal components, or spatial PCs. The spatial PCs can be paired with various analytic tools already developed in scRNA-seq studies to enable effective and novel downstream analyses for spatial transcriptomics. We illustrate the benefits of SpatialPCA through four different downstream analyses: spatial transcriptomics visualization, spatial domain detection, spatial trajectory inference on the tissue, and high-resolution spatial map reconstruction. SpatialPCA is implemented as an $\mathrm{R}$ package, freely available at www.xzlab.org/software.html.

\section{Simulations}

We performed comprehensive and realistic simulations to evaluate the performance of SpatialPCA and compare it with several other methods (Figure 1b). The compared methods in simulations include two spatial domain detection methods (BayesSpace ${ }^{29}$ and $\mathrm{SpaGCN}^{30}$ ) and two dimension reduction methods previously used for spatial transcriptomics ( $\mathrm{PCA}^{24 ; 32}$ and non-negative matrix factorization, $\mathrm{NMF}^{25}$ ). The simulation details are provided in the Methods section. Briefly, we obtained the cortex tissue from the DLPFC data, segmented it into four cortical layers, specified a distinct cell type composition of four cell types on each layer, and obtained 10,000 single cell locations on the tissue. In parallel, we simulated expression counts for 5,000 genes and 10,000 single cells from four cell types based on a separate scRNA-seq data. We then assigned the simulated cells onto the single cell locations of the cortex based on the specified cell type composition in each cortical layer to create a spatial transcriptomics data at single-cell resolution. With the single-cell resolution spatial transcriptomics, we create additional spot-level spatial transcriptomics data at different regional resolutions by merging the expression measurements of single cells into pre-defined spots on the tissue. The simulated spatial transcriptomics resemble the real data and share similar mean-variance relationship across genes (Figure S1C). For each simulated spatial transcriptomics, we then applied SpatialPCA and the other methods to perform spatial domain detection. We evaluated accuracy of different methods by comparing the detected spatial domains with the four underlying cortical layers that are served as the ground truth. We considered four simulation scenarios that cover a range of cell type compositions, with three simulation settings per scenario in the spot-level resolution spatial transcriptomics. We performed 10 simulation replicates for each setting.

In the simulated single-cell resolution spatial transcriptomics, SpatialPCA outperforms the other methods for tissue domain detection. Specifically, the median adjusted Rand index (ARI) by 
SpatialPCA across simulation replicates is $0.975,0.877,0.931$, and 0.439 for the four scenarios, respectively (Figure $1 \mathrm{~b}$ and Table S1). SpaGCN works well in scenario I when there is one dominant cell type in each spatial domain, but its performance decays in other scenarios when multiple dominant cell types are present in each spatial domain (median ARI=0.883, 0.277, 0.412, 0.091 for the four scenarios). BayesSpace does not perform as well as SpatialPCA or SpaGCN (median ARI $=0.646,0.225,0.286,0.075$ for the four scenarios), presumably because BayesSpace is specifically designed for 10X ST and Visium data and thus may not be well suited for analyzing single-cell resolution spatial transcriptomics. PCA and NMF perform similarly as BayesSpace, which is not surprising as BayesSpace does not make use of spatial information in single-cell resolution data and effectively becomes PCA there. Besides accurately detecting spatial domains, the detected spatial domains from SpatialPCA are spatially continuous and smooth, indicated by low local inverse Simpson index (LISI) score, percentage of abnormal spots (PAS) score, and spatial chaos score (CHAOS) score, more so than the other methods in all scenarios (Figure S2). The ability of SpatialPCA in clustering cells into tissue domains is in direct contrast to PCA and NMF, which are effective in clustering cells into cell types (Figure S1B). Indeed, methods that accurately cluster cells into spatial domains (e.g. SpatialPCA and SpaGCN) generally do not cluster cells into cell types well, and vice versa, highlighting the distinct goals of cell type clustering and spatial domain detection (Figure S1A and S1B). Finally, we note that while each domain detection method by default uses a different set of genes as input, all methods work almost equally well when a different set of genes are used as input (Figure S1A).

In the simulated spot-level spatial transcriptomics, SpatialPCA also outperforms the other methods in the majority of simulation settings (Figure $1 \mathrm{~b}$ and Figure S3). For example, in the simulation setting with spot diameter being 90um, the median ARI for SpatialPCA is 0.956, 0.890, 0.919 and 0.804 for the four scenarios, respectively (Figure 1b). SpaGCN again performs well in scenario 1 with one dominant cell type in each spatial domain but does not fare well in scenarios $2 \sim 4$ when multiple dominant cell types are present (median ARI $=0.915,0.214,0.292$, and 0.004 for the four scenarios). BayesSpace performs slightly worse than SpaGCN in scenario 1 but better in scenarios 2 4 (median ARI $=0.807,0.432,0.615,0.702$ for the four scenarios). PCA and NMF, on the other hand, do not perform well in all scenarios (Table S2). Besides accurately detecting spatial domains, the detected spatial domains from both SpatialPCA and BayesSpace are continuous and smooth, indicated by low LISI (Table S3), CHAOS (Table S4) and PAS (Table S5) scores, more so than the other methods in all settings.

\section{Human dorsolateral prefrontal cortex data by Visium}

We applied SpatialPCA and the other methods to analyze four published datasets obtained using different spatial transcriptomics technologies (Methods). The four datasets include a human dorsolateral prefrontal cortex data generated by Visium from 10x Genomics, a cerebellum data generated by Slide-seq, and a hippocampus data generated from Slide-seq V2, a HER2-positive breast tumor data generated by spatial transcriptomics from 10x Genomics. Besides SpatialPCA, we also examined the performance of BayesSpace, SpaGCN, stLearn, PCA, and NMF for spatial domain detection, and stLearn, PCA and NMF for trajectory inference. Among these methods, we were unable to apply BayesSpace to the Slide-seq V2 data due to the large data size and heavy computational burden. In addition, we only applied stLearn to DLPFC due to a lack of H\&E image 
in the Slide-seq and Slide-seq V2 data, and a lack of JSON file in the HER2-positive breast tumor data.

First, we analyzed the human dorsolateral prefrontal cortex data ${ }^{33}$ (Figure 2), which contains twelve samples with an average of 3,973 spots. We used sample 151673 as a main example which contains expression measurement of 33,538 genes on 3,639 spots. We first performed dimension reduction on the expression data using either SpatialPCA, PCA or NMF. For each method in turn, we summarized the inferred low dimensional components into three UMAP or tSNE components and visualized the three resulting components with red/green/blue (RGB) colors in the RGB plot (Figure 2c). The resulting RGB plots are not sensitive to the scaling of the input data (Figures S4A-S4B). The RGB plot from SpatialPCA displays laminar organization of the cortex ${ }^{33}$, shows smooth color transition across neighboring spots (Figure S4C) and neighboring spatial domains (Figure S4D). The spatial PCs are highly predictive of the cortical structures annotated by experts based on cytoarchitecture in the original study (median pseudo $\mathrm{R}^{2}=0.90$ ). In contrast, the lowdimensional components from PCA (median pseudo $\mathrm{R}^{2}=0.756$ ) and NMF (median pseudo $\mathrm{R}^{2}$ $=0.73$ ) are less predictive of the known cortical structures as compared to the spatial PCs (Figure 2f). Clustering based on the spatial PCs identified seven spatial domains that correspond to the annotated cortical layers 1 through 6 and white matter (Figure 2a). Such clustering results are robust with respect to the number of spatial genes, number of spatial PCs, the number of clusters specified, the kernel matrix used for modeling spatial correlation, as well as the clustering methods (Figure S5). The identified layers by SpatialPCA are enriched with known layer marker genes including CXCL14 (layer 2), SV2C ( layer 3), HTR2C (layer 5), and NR4A2 (layer 6/6b) ${ }^{34}$ (Figure $2 \mathrm{~b}$ ), and depict the annotated spatial domains more accurately (median ARI=0.542) than BayesSpace (ARI: median=0.438), SpaGCN (ARI: median=0.443), stLearn (ARI: median=0.470), PCA (ARI: median=0.358) and NMF (ARI: median=0.366; Figure 2d, S6-S7, and Table S6). In addition, the spatial domains detected by both SpatialPCA (median LISI=1.202; median $\mathrm{CHAOS}=0.059$; median $\mathrm{PAS}=0.02$ ) and BayesSpace (median LISI=1.234; median $\mathrm{CHAOS}=0.060$; median $\mathrm{PAS}=0.032$ ) are spatially continuous and smooth, more so than that detected by the other methods (SpaGCN: median LISI $=1.342$, CHAOS $=0.060$, PAS $=0.098$; stLearn: LISI $=1.468$, CHAOS $=0.065$, PAS $=0.175$; PCA: LISI $=1.782$, CHAOS $=0.065$, PAS $=0.28$; and NMF: LISI=1.692, CHAOS=0.066, PAS=0.27; Figure 2e and S6). SpatialPCA can be easily extended to make use of histology information as the features extracted from H\&E images can be incorporated as pseudo-dimension in the distance matrix. However, the histology feature vectors are not highly predictive for the pathologist annotations (median pseudo $\mathrm{R}^{2}=0.220$ across 12 tissue sections). Consequently, when histological feature extracted from SpaGCN were included in SpatialPCA, we observed a slightly decrease in spatial clustering performance (median ARI $=0.539$, Figures S30A).

We performed trajectory inference using the spatial PCs and detected one trajectory on the tissue (Figure S8A). The detected trajectory projects from inner to outer layers and captures the wellknown "inside-out" pattern of corticogenesis: new neurons are born in the ventricular zone, migrate along the radial glia fibers in a vertical fashion towards the marginal zone on the outskirt of the cortex, and pass the old neurons in the existing layers to form the new cortical layers ${ }^{35-37}$. In contrast, the trajectories inferred based on the low-dimensional components of PCA and NMF point towards almost random directions on the tissue. We also performed trajectory inference using stLearn, which detected multiple trajectories projecting from the white matter region to different 
cortical layers (Figure S8E) with spot level trajectories oriented towards almost random directions on the tissue (Figure S8B). We further examined genes that are associated with the inferred pseudotime (Figure S25 and Table S7) and found that the pseudo-time associated genes by SpatialPCA are highly enriched in synapse signaling, neuron projection and neuron development pathways. The identified pseudo-time associated genes highlight the importance of development and signaling of neurons across the cortical layers.

\section{Mouse cerebellum data by Slide-seq}

Next, we analyzed the mouse cerebellum data ${ }^{2}$, which contains 18,671 genes measured on 25,551 locations (Figure 3). The RGB plot from SpatialPCA displays a clear regional segregation on the tissue, more clearly than the RGB plots from PCA or NMF (Figure S9A-S9B). The resulting RGB plots are not sensitive to the scaling of the input data. Clustering based on the spatial PCs categorizes the cerebellum into eight distinct spatial domains that are consistent with the known anatomic structure of the cerebellum. These detected spatial domains include three sublayers of the granule cell layer (GCL), Purkinje cell layer, molecular layer, cerebellum nucleus, white matter, and choroid plexus (Figure 3). The clustering results from SpatialPCA are robust with respect to the number of spatial PCs used, the input gene set, the number of clusters, and the kernel matrix used for modeling spatial correlation (Figure S10). In addition, the spatial domains detected by SpatialPCA are spatially continuous and smooth (median LISI=1.404, CHAOS=0.016, PAS $=0.104$ ), more so than that detected by the other methods (BayesSpace: median LISI=3.489, $\mathrm{CHAOS}=0.028, \mathrm{PAS}=0.781$; SpaGCN: median LISI=2.157, CHAOS=0.021, PAS=0.359; PCA: median LISI $=3.166$, CHAOS $=0.026, \mathrm{PAS}=0.528$; NMF: median LISI $=3.877$, CHAOS $=0.032$, $\mathrm{PAS}=0.845$; Figure $3 \mathrm{c}, 3 \mathrm{f}$ and $\mathrm{S} 9 \mathrm{C}$ ).

A careful examination of the spatial domains detected by SpatialPCA and marker gene expression leads to two important observations. First, SpatialPCA identified an important spatial domain, choroid plexus, that is missed by the other methods (Figure 3b). Cst3, a marker gene for choroid plexus $^{38}$, is clearly enriched in the choroid plexus identified by SpatialPCA. Cst 3 encodes the cystatin $\mathrm{C}$ protein, which is secreted primarily from the choroid plexus into the cerebrospinal fluid $^{39}$. Second, unlike the other methods, the three sublayers of the GCL detected by SpatialPCA display expected spatial localization and transcriptomic profiles (Figure 3d). Specifically, the outer GCL sublayer is adjacent to the Purkinje cell layer and is enriched with the marker gene Otx2 (Figure 3g). Otx2 encodes orthodenticle homeobox 2, a transcription factor that shapes the morphogenesis of cerebellum through controlling the proliferation of postnatal granule cell precursors $^{40}$. The middle GCL sublayer is enriched with the marker gene $C b \ln 3$, which is predominantly expressed in mature granule cells that have ceased division and finished migration into the $\mathrm{GCL}^{41}$. CBLN3 forms a heteromeric complex with CBLN1 in the early postnatal stage and participates in CBLN-mediated synaptic development and function in the cerebellum ${ }^{42-44}$. The inner GCL sublayer is adjacent to the white matter and is enriched with the marker gene Eomes. Eomes encodes eomesodermin, also known as T-box brain protein 2 (TBR2), which is an important transcription factor expressed in unipolar brush cells, a type of glutamatergic interneurons in $\mathrm{GCL}^{45-47}$. TBR2 is expressed in the nuclei of a subset of interneurons in the internal granular layer in adult mouse cerebellum ${ }^{45}$ and these interneurons amplify inputs from vestibular ganglia and nuclei by spreading and prolonging excitation within the internal granular layer ${ }^{45}$. The correct 
identification of the choroid plexus and three GCL sublayers characterized by distinct transcriptomic profiles highlights the utility of SpatialPCA in revealing the transcriptomic and functional basis of fine-grained cerebellum structures.

We further examined the transcriptomic profile and cell type compositions on the tissue regions detected by SpatialPCA. First, we performed DE analysis to identify regional specific genes (Table S9) and found them to be highly enriched in the pathways of neural nucleus development and synaptic signaling (Figure S27). The enriched pathways are critical for information processing and neural development in the cerebellar cortex ${ }^{48 ; 49}$. Second, we performed deconvolution analysis to infer the cell type composition on the detected spatial domains. As expected ${ }^{50 ; 51}$, we found that the detected Purkinje layer is enriched with Bergmann and Purkinje cells; the granule cell layer is enriched with granule cells; the white matter region is enriched with oligodendrocytes; and the cerebellum nucleus is enriched with interneurons (Figure 3e). The Purkinje layer detected by SpaGCN and BayesSpace is also enriched with Purkinje cells, though it is not as well segregated from the granule and molecular layers as in SpatialPCA (Figure S11 and 3f). In contrast, clusters inferred by NMF are dominated by granule cells while each PCA cluster is generally dominated by one cell type, supporting the utility of PCA in segregating cell types rather than spatial domains as demonstrated in simulations. We also examined the distribution pattern of representative cell types in the anatomic tissue structures. We reason that, if an anatomic tissue structure is correctly depicted, then its representative cell type should be enriched in this spatial domain. In SpatialPCA and SpaGCN, the Bergmann cells are enriched in Purkinje layer as expected. However, in PCA, the Bergmann glia cells are wrongly clustered into the region of molecular layer which is adjacent to the Purkinje layer. In SpaGCN, the choroid plexus region is not detectable, such that the choroid plexus cells are distributed across multiple regions (Figure $3 \mathrm{~h}$ and S12). We did not examine the representative cell type distributions in BayesSpace and NMF because these two methods cannot detect obvious spatial domains with clear boundaries.

\section{Mouse hippocampus data by Slide-seq V2}

Next, we analyzed the mouse hippocampus data ${ }^{52}$, which contains 23,264 genes measured on 53,208 locations (Figure 4). Consistent with the previous datasets, the RGB plot from SpatialPCA displays a clear regional segregation of the tissue, more clearly than the RGB plots from PCA or NMF (Figure S13). The resulting RGB plots are not sensitive to the scaling of the input data. Clustering based on the spatial PCs categorizes the hippocampus into fourteen distinct spatial domains that are consistent with the known anatomic structure of the hippocampus. These detected spatial domains include cortical layers (layer 4, 5, and 6), corpus callosum, CA1, CA3, dentate gyrus, hippocampus regions (so/sr, slm, so), thalamus regions (subregion 1, 2, and 3), and the third ventricle (Figure 4a). The clustering results from SpatialPCA are robust with respect to the number of spatial PCs used, the input gene set, and the number of clusters (Figure S14). In contrast, clusters from SpaGCN, or clustering based on the low dimensional components from PCA and NMF do not reveal clear segregation of spatial domains (Figure 4f). Importantly, SpatialPCA also correctly detected the expected three cortical layers and three thalamus subregions while the other methods did not. In addition, the spatial domains detected by SpatialPCA are continuous and smooth (median LISI=1.043, CHAOS=0.013, PAS=0.036), more so than those detected by the other methods (SpaGCN: median LISI=2.014, CHAOS=0.016, PAS=0.031; PCA: median LISI=2.322, 

and $\mathrm{S} 13 \mathrm{C})$.

We carefully examined the transcriptomic profile and cell type compositions in the spatial domains detected by SpatialPCA. First, we performed DE analysis to identify regional specific genes (Table S10) and found them to be highly enriched in the pathways of cellular components of synapse as well as synapse signaling (Figure S28). Second, we performed deconvolution analysis to infer the cell type composition on the detected spatial domains. As expected, we found that the detected CA1 region is enriched with CA1 principal cells; the CA3 region is enriched with CA3 principal cells; the dentate gyrus region is enriched with dentate principal cells; the third ventricle region is enriched with choroid plexus cells; and the cerebellum nucleus is enriched with interneurons (Figure 4b). SpaGCN, PCA, and NMF were not able to distinguish between CA1 and CA3, as both regions are enriched with CA1 and CA3 principal cells (Figure S15). We then examined the distribution pattern of representative cell types in the anatomic tissue structures. In SpatialPCA, the entorhinal cortex cells are enriched in the three detected cortical layers as expected. However, SpaGCN and PCA are not able to delineate the cortical layers, and the entorhinal cortex cells are enriched in clusters without continuous shape or boundaries (Figure S16). We did not compare representative cell type distributions in NMF because it cannot detect obvious spatial domains with clear boundaries. Third, the spatial domains detected by SpatialPCA allowed us to identify region specific markers that are not identified by other methods. For example, only SpatialPCA detected the enrichment of Itpka, Igfbp4 and Map4 in the CA1 region of the hippocampus, consistent with their known enrichment pattern in the hippocampus pyramidal neurons ${ }^{53-55}$ (Figure 4e). Other marker genes that are highly expressed in the substructures of the hippocampus are shown in Figure 4d.

We performed trajectory inference on the cortical layers 4-6 using spatial PCs and identified one trajectory projecting from layer 6 towards 4 (Figure S17A), which is again consistent with corticogenesis $^{35-37}$. In contrast, the pseudotime values inferred based on the low-dimensional components of PCA and NMF are intermingled across layers (Figure S17B-S17C). We further examined genes that are associated with the inferred pseudo-time by SpatialPCA. We found that the pseudo-time associated genes are highly enriched in neuronal pathways such as synapse pathways and neurodegeneration diseases (Figure S25 and Table S7), highlighting the functional importance of the trajectory inferred by SpatialPCA.

\section{HER2 tumor data by spatial transcriptomics (ST)}

Finally, we analyzed the HER2-positive breast tumor data ${ }^{56 ;} 57$, which contains 15,030 genes measured on 613 spatial locations (Figure 5). Consistent with previous datasets, the RGB plot by SpatialPCA clearly segregates the tissue into multiple spatial domains, with the neighboring locations sharing more similar colors than those that are far away (Figure S18A-S18B). The resulting RGB plots are not sensitive to the scaling of the input data (Figure S18A-S18B). The top spatial PCs altogether are highly predictive of the tissue regional annotations based on the histological image obtained by pathologists in the original study (pseudo $\mathrm{R}^{2}=0.779$ ), much more so than the low dimensional components extracted from PCA (pseudo $\mathrm{R}^{2}=0.615$ ), NMF (pseudo $\mathrm{R}^{2}=0.541$ ); Figure 5d). While the correlation between spatial PCs and regular PCs are high in this 
data (the absolute Pearson's correlation between the top five spatial PCs and the top regular PCs is $0.96,0.93,0.94,0.91$ and 0.83), the spatial PCs still contain more spatial information than the regular PCs. The Moran's I of the top five spatial PCs are on average $31.6 \%$ higher than that of the corresponding regular PCs (range $=20 \%-67 \%$ ). Indeed, clustering based on the spatial PCs categorizes the tissue into seven spatial domains that are consistent with the regional annotations obtained by pathologists ( $A R I=0.431$, Figure $5 \mathrm{c}$ ). The seven detected spatial domains include normal glands, cancer region, cancer surrounding region, fibrous tissue near tumor, immune cell region, fat tissue, and fibrous tissue near normal gland (Figure 5b). The spatial domains detected by SpatialPCA are more accurate than that by the other two dimensional reduction methods (PCA: $\mathrm{ARI}=0.342 ; \mathrm{NMF}: \mathrm{ARI}=0.251$ ) and that by the two spatial domain detection methods (BayesSpace: $\mathrm{ARI}=0.416$; SpaGCN: ARI=0.282). The SpatialPCA detected a tumor immediate surrounding layer that was not observed in the original study, which highlights the ability of SpatialPCA in detecting fine grained transcriptomic changes that underlie the structural and spatial organization of carcinogenesis. The clustering results from SpatialPCA are robust with respect to the number of spatial PCs used, the input gene set, and the kernel matrix used for modeling spatial correlation (Figure S19). In addition, the spatial domains detected by SpatialPCA (median LISI=1.959, $\mathrm{CHAOS}=0.137, \mathrm{PAS}=0.26$ ) and BayesSpace are continuous and smooth (median LISI $=1.7$, $\mathrm{CHAOS}=0.138, \mathrm{PAS}=0.171$ ), more so than those detected by the other methods (SpaGCN: median LISI $=2.437$, CHAOS $=0.148$, PAS $=0.325$; PCA: median LISI=1.942, CHAOS=0.148, PAS $=0.354$; NMF: median LISI=1.724, CHAOS=0.153, PAS $=0.341$; Figure $\mathrm{S} 22 \mathrm{G}$ ). Similar to the application in DLPFC, the histological characteristic vector in the ST tumor data is not that predictive of the pathologist annotations (pseudo $\mathrm{R}^{2}=0.258$ ). Consequently, when histological information were included in SpatialPCA, we observed decreased accuracy for spatial domain detection (ARI=0.254, Figure S30C).

We characterized the transcriptomic and cell compositional properties of the spatial domains detected by SpatialPCA through two additional analyses. First, we identified genes that are specifically expressed in different spatial domains through differential expression (DE) analysis (Table S11). We found that the regional specific genes detected by SpatialPCA are highly enriched in pathways of immune response and biological adhesion (Figure S29). Second, we carefully examined the cell type compositions in each spatial domain through cell type deconvolution (Figure 5g and S23-S24). We used two scRNA-seq reference panels for deconvolution. The first scRNA-seq reference panel consists of multiple immune to help understand the spatial and compositional organization of immune cell types, which is key for characterizing the effectiveness of immune response ${ }^{58 ; 59}$. We found that the detected tumor region contains a higher fraction of mast cells and regulatory $\mathrm{T}$ cells than the other regions (Figure $5 \mathrm{~g}$ ), consistent with the function of these two cell types in promoting cancer progression. In particular, mast cells can enhance blood and lymphatic vessel formation ${ }^{60 ; 61}$ while regulatory $\mathrm{T}$ cells can suppress antitumor immune responses ${ }^{62}$. Understanding the spatial and compositional organization of different types of tumorinfiltrating immune cells can help characterize the effectiveness of immune response. The second scRNA-seq reference panel consists of both immune and cancer cells. In the analysis, we found that the detected tumor region mainly contains epithelial basal cells and epithelial basal cycling cells, which are cancer cells and cancer cells with high proliferation, respectively ${ }^{63}$. The detected tumor surrounding region is mainly composed by cancer cells with high proliferation, along with some B cells and T cells. The second deconvolution results are largely consistent with first results for the nontumor regions (Figure S24). For example, the immune region detected by SpatialPCA 
434 consists primarily of B cells and T cells, regardless of whether we include cancer cells in the reference data $(20 \% \mathrm{~B}$ cells and $36 \% \mathrm{~T}$ cells) or not (13\% B cells and $35 \% \mathrm{~T}$ cells). In PCA and $\mathrm{NMF}$, the detected tumor regions are not as smooth and continuous as SpatialPCA. In addition, PCA and NMF inferred the original annotated tumor region to consist of two or more clusters while not being able to detect the fat tissue region. Importantly, we found that the detected immune cell region resembles a tertiary lymphoid structure (TLS) ${ }^{64 ; 65}$ with multiple unique features of TLS: the region is located near the tumor; the region primarily contains $\mathrm{T}$ cells and B cells, all of which are key cell types in $\mathrm{TLS}^{64 ; 66-68}$; the region is enriched with chemokine signature genes such as CCL19 and CCL21 and T follicular helper cell signature genes such as CXCL13 and TIGIT (Figure $5 \mathrm{~h}$ ), all of which are marker genes associated with TLS neogenesis ${ }^{64}$. TLS is an ectopic lymphoid organ developed in non-lymphoid tissues that generate and regulate anti-tumor defenses. The detection of TLS is predictive of the treatment outcome in HER2-positive tumors ${ }^{69}$ and highlights the potential of SpatialPCA in understanding antitumor immune response and future prediction of tumor outcome.

The accurate tissue domains detected by SpatialPCA allowed us to identify multiple regionspecific genes that are missed by the other methods. For example, we found that TRAF2 is enriched in the tumor surrounding region detected by SpatialPCA while DDX54 is enriched in the immune region detected by SpatialPCA (Figure 5e); both enrichment patterns are not identified by the other methods. TRAF2 is associated with breast cancer progression and metastasis ${ }^{70}$, is a member of the tumor necrosis factor receptor-associated factor family of intracellular signal transduction proteins, and is a critical mediator of both the activation of NF- $\kappa \mathrm{B}$ and MAPK pathway ${ }^{71}$. The enrichment of TRAF2 in the tumor surrounding region suggests the progression of tumor cells in invading and penetrating the surrounding tissues, potentially aiding in the prognosis of cancer stage. $D D X 54$, on the other hand, encodes protein that regulates immediate-to-early DNA damage response ${ }^{72}$. Upon treatment by exposing human breast carcinoma cells to ionizing radiation, the $D D X 54$ gene interacts with pre-mRNAs that harbor introns with weak acceptor splice sites, resulting in lower intron retention and higher survival in patients ${ }^{72}$. The enrichment of $D D X 54$ in response to DNA damage in the immune regions reflect its potential role in the treatment of human breast cancer patients.

We performed trajectory inference on tumor and tumor adjacent regions to investigate how the tissue locations are connected to one another in tumorigenesis. Spatial trajectory inference based on spatial PCs identified one trajectory pointing from tumor region towards tumor surrounding region and further towards normal tissues (Figure 5f). We further examined genes that are associated with the inferred pseudo-time (Figure S25 and Table S7). We found that the pseudotime associated genes are highly enriched in RNA binding, chromatin binding, and tissue morphogenesis pathways, highlighting their importance in cancer progression, tumor invasiveness and metastasis ${ }^{73 ; 74}$. 


\section{Discussion}

474

We have presented SpatialPCA, a spatially aware dimension reduction method that is tailored for spatial transcriptomics. SpatialPCA explicitly models the spatial correlation structure in the latent space during dimension reduction and preserves the neighborhood similarity in the original data onto the low-dimensional manifold. As a result, the low-dimensional components of SpatialPCA contain valuable spatial correlation information and can be paired with existing scRNA-seq analysis tools to enable various downstream analysis of spatial transcriptomics. In addition, SpatialPCA relies on a data generative model to accommodate spatial correlation and can thus be used to impute the low dimensional components on new and unmeasured tissue locations, enabling the construction of a refined spatial map with increased spatial resolution. SpatialPCA is computationally efficient, easily scalable to spatial transcriptomics with tens of thousands of spatial locations and thousands of genes (Table S12). We have illustrated the benefits of SpatialPCA for spatial transcriptomics visualization, spatial domain detection, trajectory inference on the tissue, as well as high-resolution spatial map construction.

While we have primarily focused on spatial domain detection and trajectory inference on the tissue, we note that the modeling framework of SpatialPCA also allows us to impute low dimensional components and gene expression levels on new tissue locations, facilitating the construction of a high-resolution spatial map on the tissue (details in Methods). Because of the data generative nature of SpatialPCA and its explicit modeling of spatial correlation, SpatialPCA can be used to construct the high-resolution spatial map for any spatial transcriptomics technologies with any arbitrarily high resolution -- both these features are in direct contrast to BayesSpace, which can only enhance ST or 10x Visium data with a fixed resolution of either five or nine times higher than that of the original. We applied SpatialPCA to construct a high-resolution map in the lowresolution ST tumor data and found that the constructed high-resolution map displays continuous and spatially smooth pattern, more so than that produced by BayesSpace (Figure S20). Clustering based on the high-resolution spatial map also pinpoints precise boundaries between tissue regions and refines a thin layer of tumor surrounding region immediately outside both the tumor and immune regions.

We have primarily focused on analyzing normalized data for dimension reduction. The raw gene expression measurements from spatial transcriptomics, however, are obtained in the form of counts $^{75}$ : they are collected either as the number of barcoded mRNA for a given transcript in each single cell through smFISH based technologies or as the number of sequencing reads mapped to a given gene on each tissue location through sequencing based spatial technologies. Analyzing normalized expression data from spatial transcriptomics can be suboptimal as this approach fails to account for the mean-variance relationship existed in raw counts, leading to a potential loss of inference accuracy and subsequent loss of analysis power. Indeed, similar loss of power has been well documented for methods that only analyze normalized data in many other omics sequencing studies $^{76-79}$ as well as in spatial transcriptomics ${ }^{75}$. Consequently, many recently developed dimension reduction methods for scRNA-seq studies have chosen to directly model raw count data, which have resulted in improvement in inference accuracy ${ }^{21-23}$. In principle, SpatialPCA can be extended to model raw count data from spatial transcriptomics based on the generalized linear model framework, with an additional zero component to model the potential zero inflation that might be encountered in certain spatial transcriptomic technologies. Such extension of SpatialPCA, 
however, will likely incur substantial increase in computational cost, along with potentially numerical instability issues associated with optimizing the likelihood function in the presence of sparse counts ${ }^{80 ; 81}$. Extending SpatialPCA towards effective modeling of count data while keeping computational cost and numerical stability in check will be an important future research direction.

We have primarily focused on performing spatially aware dimension reduction based on the probabilistic version of PCA. PCA is a linear dimension reduction method that effectively expresses the low dimensional components as a linear function of the input matrix. Despite its simplicity, dimension reduction based on PCA is surprisingly effective and facilitates many downstream analytic tasks in scRNA-seq studies ${ }^{17}$. Indeed, PCA is implemented in commonly applied scRNA-seq tools such as Seurat ${ }^{24}$, SCANPY ${ }^{82}$ and Cell Ranger R Kit ${ }^{83}$, for downstream data visualization, clustering analysis, or trajectory inference. Certainly, dimension reduction based on linearity likely only captures the first order relationship between the original data and the reduced manifold and may not be effective in capturing all complex biological signals contained in the input genomic data. To further improve the effectiveness of dimension reduction and capture non-linear signals in the expression matrix, several non-linear dimension reduction methods have been recently developed for scRNA-seq. These non-linear methods are often based on deep neural networks and can flexibly model the non-linear relationship between the expression matrix and the extracted low-dimensional components ${ }^{84-87}$. Extending SpatialPCA towards non-linear modeling based on the deep learning framework for spatial transcriptomics is another important future direction.

Finally, we note that the main idea of incorporating spatial correlation information into PCA has a long-standing history in geographics and genetics. In geographics, PCA has been commonly applied for dimension reduction in geographical datasets ${ }^{88}$ and has been previously extended to a geographically weighted version that performs local PCA at each spatial location while accommodating the local neighboring structure ${ }^{89}$. In genetics, a spatial version of PCA was previously developed to incorporate the autocorrelation structure measured by Moran's I into PCA for the identification of cryptic spatial patterns of genetic variability ${ }^{90}$. Our SpatialPCA effectively extends these ideas into a formal data generative model with a maximum likelihood inference framework. Compared to these early approaches, SpatialPCA performs dimension reduction at the global instead of local level, automatically infers the contribution of spatial correlation in determining the variation in the low dimensional components, and is capable of imputing low dimensional components in new and unmeasured spatial locations. Because of these modeling advantages, examining the utility of SpatialPCA in applications beyond spatial transcriptomics 554 may benefit other research fields. 


\section{SpatialPCA Overview}

We consider a spatial transcriptomics study that collects gene expression measurements for $m$ genes on $n$ spatial locations of a tissue. These locations have known spatial coordinates that are recorded during the experiment. We denote $\boldsymbol{s}_{\boldsymbol{i}}$ as the $k$-vector of spatial coordinates for $i^{\prime}$ th location, with $i \in(1, \ldots, n)$. Depending on the spatial transcriptomics technology, the spatial coordinates vary continuously over either a two-dimensional space $\left(k=2 ; s_{i}=\left(s_{i 1}, s_{i 2}\right) \in R^{2}\right)$ or a three-dimensional space $\left(k=3 ; \boldsymbol{s}_{\boldsymbol{i}}=\left(s_{i 1}, s_{i 2}, s_{i 3}\right) \in R^{3}\right)$. We denote $\boldsymbol{Y}$ as the $m \times n$ gene expression matrix measured in the study. The $i j$ 'th element of $\boldsymbol{Y}, y_{i j}\left(\boldsymbol{s}_{i}\right)$, represents the gene expression measurement for $j$ 'th gene on $i$ 'th location. Following ${ }^{83 ; 91 ; 92}$, we assume that the expression measurements have already been normalized through variance stabilizing transformation and further scaled for each gene to have zero mean and unit standard deviation.

Our goal is to perform dimension reduction on the gene expression matrix and infer a $d \times n$ factor matrix $\boldsymbol{Z}$ that represents a low-dimensional embedding of $\boldsymbol{Y}$. The factor matrix $\boldsymbol{Z}$ contains $d$ factors, and its $l$ 'th row, $\boldsymbol{Z}_{l}$, is an $n$-vector that represents the $l^{\prime}$ th factor values across $n$ locations. For dimension reduction, we consider the following latent factor model

$$
\boldsymbol{Y}=(\boldsymbol{X} \boldsymbol{B})^{T}+\boldsymbol{W} \boldsymbol{Z}+\boldsymbol{E},
$$

where $\boldsymbol{X}$ is an $n \times q$ matrix of covariates, which contains a column of ones as the intercept along with $(q-1)$ other potential covariates; $\boldsymbol{B}$ is a $q \times m$ matrix of corresponding coefficients; $\boldsymbol{W}$ is a $m \times d$ factor loading matrix; and $\boldsymbol{E}$ is an $m \times n$ matrix of residual errors. In the present study, we only include the intercept for all analyses and thus $q=1$. We assume that the $i j$ 'th element of $\boldsymbol{E}, \boldsymbol{E}_{i j}$, follows an independent normal distribution with mean zero and variance $\sigma_{0}^{2}$, or $\boldsymbol{E}_{i j} \sim N\left(0, \sigma_{0}^{2}\right)$.

The factor model in equation (1) is not yet identifiable as any rotational transformation of $\boldsymbol{W}$ and $\boldsymbol{Z}$ would lead to the same solution. Therefore, we need to place further modeling constraints on both $\boldsymbol{W}$ and $\boldsymbol{Z}$ to ensure model identifiability. For $\boldsymbol{W}$, we follow the probabilistic principal component analysis model (PPCA) ${ }^{93}$ and impose an orthonormality constraint on its columns to have $\boldsymbol{W}^{\boldsymbol{T}} \boldsymbol{W}=\boldsymbol{I}_{d}$. For $\boldsymbol{Z}$, PPCA $^{93}$ and other previous approaches ${ }^{94 ; 95}$ commonly assume element independence. That is, each element of $\boldsymbol{Z}$ is independently and identically distributed from a normal distribution $N(0,1)$. However, such element independence assumption on $\boldsymbol{Z}$ is not ideal for spatial transcriptomics. In spatial transcriptomics, the neighboring locations on a tissue often share similar composition of cell types and display similar gene expression levels. Consequently, the factor values on neighboring locations are likely similar to each other, more so than those on locations that are far away. The factor values on neighboring locations thus contain invaluable information that can be used to facilitate the inference of the factor values on the location of interest. To encourage neighborhood similarity in factor values and facilitate information sharing across neighboring locations for factor estimation, we follow ${ }^{96}$ and assume that each $\boldsymbol{Z}_{l}$. follows a multivariate normal distribution

$$
\mathbf{Z}_{l} \sim \operatorname{MVN}\left(\mathbf{0}, \boldsymbol{\Sigma}_{l}\right),
$$


where the $n \times n$ covariance matrix $\boldsymbol{\Sigma}_{l}$ models the correlation among the spatial locations and is a function of their spatial coordinates. Here, we use the Gaussian kernel to construct the covariance matrix and assume that the $\left(i, i^{\prime}\right)$ th entry of $\boldsymbol{\Sigma}_{l}$ is in the form of $\sigma_{0}^{2} \tau_{l} \boldsymbol{K}\left(\boldsymbol{s}_{i}, \boldsymbol{s}_{i \prime}\right)$, where $\boldsymbol{K}\left(\boldsymbol{s}_{i}, \boldsymbol{s}_{i^{\prime}}\right)=$ $\exp \left(-\left|\boldsymbol{s}_{i}-\boldsymbol{s}_{i},\right|^{2} / \gamma\right)$ with $\gamma$ being the bandwidth parameter and $\sigma_{0}^{2} \tau_{l}$ being a variance component that is scaled with respect to the residual error variance $\sigma_{0}^{2}$. The functional form of $\boldsymbol{\Sigma}_{l}$ is designed to induce spatial correlation of factor values on the tissue and encourage factor similarity in neighboring locations. Specifically, if two locations are close to each other, then the corresponding element in $\boldsymbol{\Sigma}_{l}$ will be large, leading to similar factor values on the two locations; and vice versa. The bandwidth parameter $\gamma$ in $\boldsymbol{\Sigma}_{l}$ determines the strength of such spatial correlation: a small $\gamma$ leads to a high spatial correlation while a large $\gamma$ leads to a low spatial correlation ${ }^{97 ;}$ 98. The variance parameter $\tau_{l}$ in $\boldsymbol{\Sigma}_{l}$, on the other hand, determines the scale of the l'th factor values: a small $\tau_{l}$ corresponds to small factor values relative to the residual errors while a large $\tau_{l}$ corresponds to large factor values relative to the residual errors. For $\gamma$, we follow previous approaches ${ }^{99 ; 100}$ and determine $\gamma$ in a sample size dependent fashion. Specifically, for a data with 613 large samples ( $>5,000)$, we use Silverman's "rule-of-thumb" bandwidth, which is defined as $0.9 \min \left(\hat{\sigma}, \frac{I Q R}{1.34}\right) n^{-\frac{1}{5}}$. Here, the bandwidth is computed for one gene at a time, where $\hat{\sigma}$ is the standard deviation of gene expression and $I Q R$ is the interquartile range of gene expression ${ }^{99}$. We obtain the median bandwidth value across all genes to serve as $\gamma$. For a data with small samples, the asymptomatic "rule-of-thumb" bandwidth is no longer applicable. Thus, we use the nonparametric Sheather \& Jones's bandwidth that is especially robust for data with small samples ${ }^{100}$. We again compute the bandwidth for each gene in turn and obtain the median value across all genes to serve as $\gamma$. For $\tau_{l}$, we follow PPCA $^{93}$ and assume scale homogeneity across factors by setting $\tau_{l}=\tau$.

With the above model specifications, we infer the factor loading matrix $\boldsymbol{W}$ and the factor matrix $\boldsymbol{Z}$, along with the hyper-parameters $\left(\tau, \sigma_{0}^{2}\right)$, through maximum likelihood based optimization. Specifically, we first integrate out both $\boldsymbol{B}$ and $\boldsymbol{Z}$ to obtain a marginal likelihood, based on which we infer $\tau, \sigma_{0}^{2}$ and $\boldsymbol{W}$ (details in the Supplementary Text). We then estimate $\boldsymbol{Z}$ by computing their posterior mean conditional on the estimated $\tau, \sigma_{0}^{2}$ and $\boldsymbol{W}$. In the algorithm, we incorporate multiple algebraic innovations to enable scalable computation and apply a low rank approximation on the kernel matrix $\boldsymbol{K}$, with a low rank $r$ to ensure that the approximate matrix captures at least $90 \%$ of 630 the variance in the original matrix in the present study. Overall, the computational time complexity of our algorithm is $O\left(t d m^{2}+r n^{2}\right)$, where $t$ represents the number of iterations used in the optimization algorithm, with memory requirement being $O\left(m n+n^{2}\right)$ (Details in Supplementary Text). To further save memory and computation time, we also provide in the software the option to calculate a sparse kernel matrix when the sample size is large. The user can determine the sparsity level of the matrix by providing a cut-off value (default 1e-20) to set every element below this value to be 0 . Because the factor model builds upon PPCA and the factor matrix $\boldsymbol{Z}$ contains crucial spatial correlation information among locations, we refer to our model as the spatial probabilistic PCA (SpatialPCA) and refer to these factors as spatial PCs. SpatialPCA is implemented in an R package, which uses an S4 object to contain the model parameters and takes 640 raw expression count data and spatial locations as input. SpatialPCA is freely available at 


\section{Downstream Analyses with Spatial PCs}

The inferred spatial PCs $\boldsymbol{Z}$ from SpatialPCA can be paired with various methods already developed in the scRNA-seq literature to enable a range of downstream applications in spatial transcriptomics. Here, we examine the use of SpatialPCA for two important analytic tasks in spatial transcriptomics: spatial domain detection and spatial trajectory inference on the tissue. Spatial domain detection aims to segment the tissue into multiple structures, domains or microenvironments, each of which is characterized by a distinct transcriptomic profile. Spatial trajectory inference, on the other hand, aims to infer the transcriptomic relationship among spatial locations and construct trajectories directly on the tissue to represent the potential developmental lineages across locations that happened in the past. To the best of our knowledge, only a handful methods have been developed for spatial domain detection ${ }^{30 ; 101}$ and no method has been published for spatial trajectory inference on the tissue. For spatial domain detection, we formulate it as a clustering problem on the inferred spatial PCs $\boldsymbol{Z}$. In particular, we apply standard scRNA-seq clustering algorithms on $\boldsymbol{Z}$ to categorize spatial locations into different spatial domains. Because $\boldsymbol{Z}$ contains critical spatial correlation information across locations, clustering based on the spatial PCs would lead to similar cluster assignment in the neighboring locations, resulting in smooth boundaries in the detected tissue structures. For spatial trajectory inference on the tissue, we apply standard scRNA-seq trajectory analysis methods on $\boldsymbol{Z}$ to infer the progression in gene expression across spatial locations. Spatial trajectory inference on $\boldsymbol{Z}$ allows us to properly account for the spatial relationship among locations, resulting in continuous trajectories with consistent directionality across neighboring locations. In the present study, we use Walktrap algorithm and Louvain algorithm for clustering analysis and slingshot ${ }^{20}$ for trajectory analysis following the recommendations of ${ }^{19 ; 29 ; 33}$. However, SpatialPCA can be potentially paired with any clustering algorithm ${ }^{17}$ or trajectory inference algorithm ${ }^{19}$ developed in the scRNA-seq literature to take advantage of their benefits.

Importantly, because SpatialPCA builds upon a data generative model that automatically infers the spatial correlation structure across tissue locations, it can also be used to construct a refined spatial map on the tissue. In particular, with the inferred spatial correlation, we can predict and impute spatial PCs on new and unmeasured locations based on the inferred spatial PCs on the measured locations. Imputing spatial PCs on new locations would allow us to obtain a spatial map with a resolution much higher than that measured in the original study. To do so, we examine one spatial PC at a time. For the l'th spatial PC, we denote $Z_{l}(s)$ as the $n$-vector of spatial PC values on the original locations $\boldsymbol{S}$ and denote $\widetilde{\boldsymbol{Z}}_{\boldsymbol{l}}(\widetilde{\boldsymbol{s}})$ as the $\tilde{n}$-vector of spatial PC values on the $\tilde{n}$ new locations $\tilde{\boldsymbol{s}}$. Based on equation (2), the $(n+\tilde{n})$-vector of $\left(\boldsymbol{Z}_{\boldsymbol{l}}(\boldsymbol{s}), \widetilde{\boldsymbol{Z}}_{\boldsymbol{l}}(\tilde{\boldsymbol{s}})\right)^{\boldsymbol{T}}$ follows a multivariate normal distribution. Consequently, we can obtain the conditional mean of $\widetilde{\boldsymbol{Z}}_{\boldsymbol{l}}(\widetilde{\boldsymbol{s}})$ given $\boldsymbol{Z}_{\boldsymbol{l}}(\boldsymbol{s})$ based on the property of multivariate normal distribution:

$$
\widetilde{Z}_{l}(\widetilde{\boldsymbol{s}})=\widetilde{\boldsymbol{\Sigma}}_{l}^{\mathrm{T}}(\widetilde{\boldsymbol{s}}, \boldsymbol{s}) \Sigma_{l}^{-1} Z_{l}(s)
$$

where $\widetilde{\boldsymbol{\Sigma}}_{l}(\widetilde{\boldsymbol{s}}, \boldsymbol{s})=\sigma_{0}^{2} \tau K(\widetilde{\boldsymbol{s}}, \widetilde{\boldsymbol{s}})$ is an $\tilde{n}$ by $\mathrm{n}$ covariance matrix measuring the spatial correlation between the new and measured locations. We use the conditional mean in equation (3) to serve as the imputed spatial PCs on the new locations.

The imputed PCs on the new locations also allow us to directly impute the gene expression levels for individual genes on the new locations. Specifically, we calculate the posterior mean of gene 
expression as the product of the estimated loading matrix $\widetilde{W}$ and the high resolution predicted spatial PCs $\widetilde{Z}_{l}(\widetilde{\boldsymbol{s}})$, with $\widetilde{\boldsymbol{Y}}=\widetilde{\boldsymbol{W}} \widetilde{\boldsymbol{Z}}_{\boldsymbol{l}}(\widetilde{\boldsymbol{s}})$.

\section{Simulations}

We performed simulations to evaluate the performance of our method and compare it with the other methods. To do so, we first obtained the cortex tissue from the DLPFC data (sample id 151673) and manually segmented the tissue into four cortical layers through Adobe Illustrator as illustrated in Figure 1a. We exported the illustrative figure as a 1100 pixel by 984 pixel image in JEPG format and extracted the four cortical layer labels for each pixel based on the RGB values. We then randomly sampled 10,000 pixels from the image to serve as the single cell locations and extracted their $\mathrm{x} / \mathrm{y}$ coordinates. We assumed that these single cells belong to four different cell types, with distinct cell type composition in each cortical layer. In particular, we set the cell types with the highest to lowest proportions to be 1, 2, 3, and 4 in the first layer, 2, 3, 4, 1 in the second layer, 3, 4, 1, 2 in the third layer, and 4, 1, 2, 3 in the fourth layer. We then considered four cell type composition scenarios in the simulations. In the first scenario, each layer contains one dominant cell type, with $85 \%$ of the cells belonging to the dominant cell type and $5 \%$ of the cells belong to each of the three minor cell types. In the second scenario, each layer contains two dominant cell types with equal proportion, each consisting of $45 \%$ of cells, along with two minor cell types each consisting of 5\% of cells. In the third scenario, each layer contains two major cell types with unequal proportion, with one consisting of $60 \%$ of cells and the other consisting of $30 \%$ of cells, along with two minor cell types each consisting of $5 \%$ of cells. In the fourth scenario, each layer contains three major cell types and one minor cell type, consisting of $35 \%, 30 \%, 30 \%$, or $5 \%$ of cells, respectively. In each scenario, we randomly assigned each single cell in each layer to one of the four cell types based on a multinomial distribution with parameters set to be the cell type composition in the layer. We performed 10 simulation replicates for each simulation scenario.

In parallel, we obtained a scRNA-seq data on human prefrontal cortex obtained through Smartseq2 $2^{102}$, which contains expression measurements for 24,153 genes and 2,394 cells that belong to six cell types annotated in the original study. The six cell types include astrocytes $(\mathrm{n}=76$ cells), GABAergic neurons $(n=701$ cells), microglia $(n=68)$, neuron cells $(n=1,057)$, oligodendrocyte progenitor cell (OPC; $n=117)$, and stem cells $(n=290)$. We used the cells from the neuron cells in the scRNA-seq data as a reference to simulate gene expression counts for 5,000 genes and 30,000 single cells using Splatter ${ }^{103}$. In Splatter, we used the splatEstimate function to estimate the cell type parameters in the $17 \mathrm{cRNA}$-seq data; we set de.prob=0.5; and we set group.prob $=c(0.25,0.25,0.25,0.25)$ so that the four cell types have equal probability. From the simulated cells, we randomly selected 10,000 of them with the desired cell type compositions determined by the simulation scenario described in the above paragraph and assigned these cells onto the 10,000 locations to create the single-cell resolution spatial transcriptomics. With the single-cell resolution spatial transcriptomics, we further generated spot-level spatial transcriptomics data by merging expression counts of single cells into spots. In particular, we created square grids on the tissue, treated each square grid as a subspot, and annotated the spatial domain label of each subspot based on the majority of the spatial domain labels for cells located within the subspot. We treated the spatial domain label for the subspots as ground truth for spatial clustering accuracy comparison. We merged every nine subspots into a spot following the 10X ST 
subspot layout ${ }^{29 ; 104}$ and obtained the coordinates for the spot based on the coordinates of the center subspot. We varied the length of the square grids to create three spot-level spatial transcriptomics settings with varying resolution, with spot diameter being $90 \mathrm{um}(\mathrm{n}=5,077$ spots $), 107 \mathrm{um}(\mathrm{n}=3,602)$, or 145um $(n=1,948)$; where the spot diameter for a 10X ST data is $100 \mathrm{um}$. We focused on the simulation setting with spot diameter being 90um in the main text and places the other two cases in supplementary figures.

For each simulated spatial transcriptomics, we applied SpatialPCA and the other methods to detect spatial domains on the tissue. The other methods include BayesSpace, SpaGCN, PCA and NMF. We did not compare with stLearn in the simulations as the simulations did not contain H\&E images - stLearn becomes PCA without an H\&E image. Following the recommendation of SPARK-X ${ }^{80}$, we obtained SVGs in the single cell level simulations using SPARK-X due to its efficiency in computational speed and memory cost and obtained SVGs in the spot level simulations using SPARK due to its higher statistical power.

\section{Analyzed Datasets}

We examined four public spatial transcriptomics datasets that include the followings.

DLPFC human prefrontal cortex data by Visium. We downloaded 12 human DLPFC ${ }^{33}$ tissue samples from three individuals on the Visium platform (http://spatial.libd.org/spatialLIBD/). The 12 samples measured on an average of 3,973 spots that were manually annotated to one of the six prefrontal cortex layers or white matter. We used the sample 151673 as the main analysis example, which contains expression measurement of 33,538 genes on 3,639 spots. We presented the results for the other 11 samples in the supplementary figures. In the analysis, we retained genes with nonzero expression on at least 20 spots and retained spots with non-zero expression for at least 20 genes. These filtering steps lead to a final set of 15,124 genes measured on 3,639 spots for analysis. The annotated spatial domains in sample 151673 in the original study is based on cytoarchitecture and include Layer $1(n=273)$, Layer $2(n=253)$, Layer $3(n=989)$, Layer $4(n=218)$, Layer $5(n=673)$, Layer $6(n=692)$, white matter $(n=513)$, and 28 undetermined locations. In the spatial domain detection analysis described in the following section, we excluded the undetermined locations and treated the remaining regional annotations as ground truth.

Mouse cerebellum data by Slide-seq. We obtained the Slide-seq data on mouse cerebellum from the Broad Single Cell Portal (ID SCP354)2. We used the file "Puck_180430_6", which contains 18,671 genes measured on 25,551 spatial locations. We removed mitochondrial genes and retained genes with non-zero expression level on at least 20 locations. We also retained locations with nonzero expression for at least 20 genes. These filtering steps lead to a final set of 10,515 genes on 20,982 locations for analysis. The Slide-seq data does not come with tissue domain annotations that can serve as ground truth as tissue domain detection was not performed in the original study. Therefore, we relied on the Allen Brain Atlas ${ }^{105}$ and other previous literatures ${ }^{50 ; 51 ; 106-113}$ to help determine which cluster corresponds to which tissue structure. Because of a lack of corresponding H\&E image, such annotations are approximate and cannot be used as groundtruth to evaluate spatial clustering performance. 
Mouse hippocampus data by Slide-seq V2. We obtained the Slide-seq V2 data ${ }^{52}$ from https://singlecell.broadinstitute.org/single_cell/study/SCP815/sensitive-spatial-genome-wideexpression-profiling-at-cellular-resolution\#study-summary. We used the file "Puck_200115_08", which contains 23,264 genes measured on 53,208 spatial locations. We retained genes with nonzero expression level on at least 20 locations and locations with non-zero expression for at least 20 genes. These filtering steps lead to a final set of 16,235 genes on 51,398 locations for analysis. The Slide-seq V2 data does not come with tissue domain annotations that can serve as ground truth as tissue domain detection was not performed in the original study. Therefore, we relied on the Allen Brain Atlas ${ }^{105}$ and other previous literatures ${ }^{114-117}$ to help determine which cluster corresponds to which tissue structure. Because of a lack of corresponding H\&E image, such annotations are approximate and cannot be used as groundtruth to evaluate spatial clustering performance.

HER2 tumor data by spatial transcriptomics (ST). We downloaded the HER2-positive breast tumor data collected from ST platform from https://github.com/almaan/her2st (Andersson et al. $2021^{56}$ ). We used the sample $\mathrm{H} 1$ as the main analysis example, which contains expression measurements of 15,030 genes on 613 spatial locations. The results for the other 36 samples are provided in the supplementary figures. In the analysis, we retained genes with non-zero expression on at least 20 spots and retained spots with non-zero expression for at least 20 genes. We also removed 21 genes associated with a ring pattern observed in multiple samples in the original study. These genes were confounded by technical artifacts as explained in the original study. These filtering steps lead to a final set of 10,053 genes measured on 607 spots for analysis. The ST data consists of seven spatial domains that were annotated by pathologists based on H\&E staining of the same tissue section. The seven annotated spatial domains include in situ cancer ( $\mathrm{n}=97$ spatial locations), invasive cancer $(n=90)$, breast glands $(n=39)$, adipose tissue $(n=112)$, immune infiltrate $(n=23)$, connective tissue $(n=166)$, and other spots in undetermined region $(n=80)$. In the spatial domain detection analysis described in the following section, we excluded the undetermined region and treated the remaining regional annotations as ground truth. Note that a previous study on the same ST tumor data provided a slightly different set of tissue domain annotations (Salmén et al. $\left.2018^{118}\right)$. The previous seven annotated spatial domains include fat tissue $(n=92)$, fibrous tissue $(\mathrm{n}=271)$, immune cells $(\mathrm{n}=52)$, in situ cancer DCIS $(\mathrm{n}=103)$, invasive cancer $(\mathrm{n}=31)$, necrosis $(n=11)$, and normal glands $(n=47)$. Because the results based on both sets of annotations are largely similar, we presented results based on the new Andersson et al. $2021^{56}$ annotation in the main text and provided results from the old Salmén et al. $2018^{118}$ annotation in the supplementary figures.

\section{Analysis Details}

\section{Data normalization and dimension reduction.}

For all datasets, we normalized the raw expression count matrix using the variance stabilizing transformation (VST) implemented in the Seurat function in SCTransform ${ }^{92}$. We then standardized the normalized expression values of each gene to have zero mean and unit standard deviation following $83 ; 91 ; 92$. We used the resulting standardized expression matrix as input for all dimension reduction methods that include SpatialPCA, PCA and NMF. 
For these dimension reduction methods, we performed additional gene filtering to retain a set of spatially expressed genes as input. To do so, we followed the recommendation of SPARK-X ${ }^{80}$ to use SPARK ${ }^{119}$ for SVG analysis in small datasets (ST tumor and DLPFC) due to its higher statistical power and use SPARK-X for SVG analysis in larger datasets (Slide-seq and Slide-seq V2) due to its computational efficiency. Genes are declared as significant based on an FDR threshold of 0.05 using the Benjamini-Yekutieli procedure implemented in the software. We used up to 3,000 significant SVGs as input, with 3,000, 319, 787, and 3,000 SVGs selected in the DLPFC, ST tumor, Slide-seq, and Slide-seq V2 data, respectively. We applied the three dimension reduction methods on the same standardized data matrix for the selected SVGs. In particular, we fitted PCA by applying singular value decomposition (SVD) ${ }^{120}$ and fitted NMF using the RunNMF function in STUtility package ${ }^{25 ; 121}$. Regardless of the method, we extracted the top 20 low-dimensional components for downstream analyses.

We examined the sensitivity of different methods by carrying out dimension reduction using three different sets of genes as input in the DLPFC data. The three gene sets include all genes, 3,000 top highly variable genes (HVGs), and 3,000 top SVGs. The HVGs are obtained using the FindVariableGenes function in the Seurat package. For the set of all genes, applying SpatialPCA in DLPFC incurs heavy computational burden due to its iterative eigen decomposition of a geneby-gene matrix. Therefore, we first performed PCA to extract the top 3,000 regular PCs from the set of all genes and then used them as input for SpatialPCA.

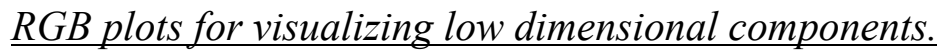

For SpatialPCA, PCA, and NMF, we summarized the inferred low dimensional components into three UMAP or tSNE components and visualized the three resulting components with $\mathrm{red} / \mathrm{green} / \mathrm{blue}$ (RGB) colors through RGB plot. For each method, we normalized the tSNE or UMAP embeddings to be in the range of 0 to 1 and used these normalized values as input for the $\mathrm{rgb}$ () function in the ggplot function to generate RGB plot. In the process, we explored the sensitivity of RGB plot to the scale of the input low dimensional components by multiplying them 10 or 20 times. On the RGB plot, we also summarized the RGB colors on each location into a vector in the form of weighted RGB values $R G B_{\text {weighted }}=\frac{R * \operatorname{var}(R)+G * \operatorname{var}(G)+B * \operatorname{var}(B)}{\operatorname{var}(R)+\operatorname{var}(G)+\operatorname{var}(B)}$, where $R, G, B$ represent vectors of the color values for the three channels; while $\operatorname{var}(\cdot)$ represents the sample variance computed across locations and serves as the weights. We scaled $R G B_{\text {weighted }}$ to have mean zero and variance one. In the DLPFC data, we examined the spatial distribution of the weighted RGB values across spatial domains. We also calculated for each spot the variance of the rescaled $R G B_{\text {weighted }}$ on the spot and its six nearest spots, where a small variance indicates spatial smoothness in neighborhood RGB values.

\section{Spatial domain detection via clustering.}

We performed clustering analysis on the low-dimensional components to cluster spatial locations. Clustering locations allows us to effectively segment the tissue into distinct spatial domains. Here, we applied two clustering algorithms: the Walktrap method and the Louvain method. For the Walktrap method, we first constructed a shared nearest neighbors (SNN) graph ${ }^{122}$ based on the low-dimensional components using the scran package ${ }^{123}$. SNN measures similarity between each pair of locations by counting the number of neighboring locations that are connected to both locations. With the SNN graph, we applied the Walktrap method using the bluster R package ${ }^{124}$ to obtain the cluster labels for locations. For the Louvain method, we first built a k-nearest neighbors 
871 (KNN) network among locations using the FNN package ${ }^{125}$. In the KNN network, each location 872 is connected to its K nearest locations in the Euclidean space. With the KNN network, we applied the Louvain community detection algorithm to cluster locations using the igraph R package ${ }^{126}$. We applied the Walktrap method to the small datasets (DLPFC and ST tumor) and due to its heavy computational burden applied the Louvain method to the other two large datasets. We set the number of spatial clusters in the DLPFC and ST tumor datasets based on the ground truth annotation and set the number of clusters to achieve the highest average Silhouette width ${ }^{127}$ in the Slide-seq data. In Slide-seq V2, we were unable to calculate the Silhouette width because the required distance matrix is too big due to the large sample size. Therefore, we set the number of clusters in Slide-seq V2 based on the number of spatial domains annotated in Allen Brain Atlas ${ }^{105}$. After clustering, we performed an additional refinement step following SpaGCN to relabel a spot based on the majority of the domain labels from its surrounding spots along with itself if the two are different (four surrounding spots in the ST data and six in the Visium data).

\section{Compared methods for spatial domain detection.}

We compared the performance of the above three dimension reduction methods with three spatial domain detection methods for detecting spatial domains on the tissue. The three examined domain detection methods include BayesSpace, SpaGCN, and stLearn.

BayesSpace ${ }^{29}$ is designed for spatial domain detection in ST or Visium data from 10x genomics. BayesSpace performs PCA on the top 2,000 HVGs and models the top 15 PCs to infer the latent cluster labels for spatial domains. BayesSpace can enhance the resolution of clustering in ST or Visium data by segmenting each spot to 6 (for Visium) or 9 subspots (for ST) and uses a Potts prior to infer the spatial cluster labels on the subspots. For fitting BayesSpace, we set the parameter nrep in spatialPreprocess to be 50,000 in DLPFC and Slide-seq data, and to be 10,000 in ST tumor data due to its small sample size following the BayesSpace tutorial. We set the number of burn in to be 1,000 in all datasets. We did not run BayesSpace on the Slide-seq V2 data because it requires too much memory $(>100 \mathrm{~Gb})$.

908

$\mathrm{SpaGCN}^{30}$ uses gene expression, spatial location and histology information as inputs to infer spatial domains on the tissue. Specifically, SpaGCN reduces the dimension of the preprocessed gene expression matrix using PCA to obtain the top 50 PCs as input. SpaGCN builds a graph to incorporate the spatial and histology information, and then uses a graph convolutional layer to reconstruct the gene expression information by aggregating spatial and histological information. In the DLPFC data, for all twelve samples, we followed the SpaGCN tutorial and provided high resolution H\&E image to SpaGCN. In ST tumor data, we used H\&E image with lower resolution downloaded from https://github.com/almaan/her2st. For Slide-seq and Slide-seq V2 data, no H\&E images is available, so we choose the "histology=False" option in SpaGCN.

stLearn ${ }^{31}$ leverages spatial location and histology information to smooth gene expression for downstream clustering and trajectory inference. stLearn uses gene expression, spatial location and histology information as inputs. We followed the default settings in the tutorial of stLearn to perform clustering analysis on the 12 samples in DLPFC. We did not apply stLearn to ST tumor data as the original data paper does not provide a JSON file that is necessary for stLearn. We also did not apply stLearn to the Slide-seq and Slide-seq V2 data as neither has H\&E information. In trajectory inference, stLearn applies a diffusion pseudotime method on the smoothed gene 
expression data and focuses on ordering the spatial clusters based on their average pseudotime within clusters. We compared the pseudotime calculated from stLearn with that from SpatialPCA in sample 151673 of the DLPFC data.

\section{Spatial clustering performance evaluation.}

For the first two datasets that come with spatial domain annotations that can serve as ground truth, we evaluated the performance of different methods in spatial domain detection by comparing the detected spatial domains with truth in two different ways. First, we used standard clustering evaluation metrics that include the adjusted rand index (ARI) ${ }^{128}$ and normalized mutual information (NMI) ${ }^{129}$. Second, we directly evaluated the information contained in the output from different methods in predicting the true spatial domains without performing explicit clustering. Specifically, we treated the true spatial domains as the outcome and fitted a multinomial regression model. For the dimension reduction methods, we treated the extracted low-dimensional components as predictors in the regression model. In the regression model, we computed the McFadden adjusted pseudo- $\mathrm{R}^{2}{ }^{130}$ to evaluate the predictive ability of the predictor variables in predicting the ground truth. A higher pseudo- $\mathrm{R}^{2}$ suggests that the method is capable of extracting informative output in predicting the true spatial domains.

For all datasets, we used another three metrics to evaluate clustering performance. First, we adopted the integration quality quantification metric used in scRNA-seq and calculated the local inverse Simpson's index (LISI) ${ }^{131}$ to quantify the clustering performance for spatial domain detection. The LISI score is the effective number of spatial domain labels represented in the local spatial neighborhood of a spot and is calculated as

$$
S=\frac{1}{\sum_{k=1}^{K} p(k)}
$$

where $p(k)$ is the probability that the spatial domain cluster label $k$ is in the local neighborhood, and $K$ is the total number of spatial domains. We calculated the LISI score using the compute_lisi function from the LISI R package with default parameters (perplexity $=30$ ). Lower LISI score indicates more homogeneous neighborhood spatial domain clusters of the spot.

Second, we adopted image segmentation performance quantification metrics in mass spectrometry imaging $^{132}$ and used spatial chaos score (CHAOS) and percentage of abnormal spots (PAS) score to quantify the clustering performance for spatial domains.

The CHAOS score measures the spatial continuity of the detected spatial domains ${ }^{132 ; 133}$. To calculate CHAOS, we first create a one-nearest-neighbor (1NN) graph for the spots in each spatial cluster by connecting each spot with its nearest neighbor. We denote $w_{i j}$ as the edge weight between spot $i$ and spot $j$, which is calculated as

$$
w_{k i j}=\left\{\begin{array}{c}
d_{i j}, \quad \text { if spot } i \text { and } j \text { are connected in the } 1 N N \text { graph in cluster } k \\
0, \text { otherwise }
\end{array}\right.
$$

where $d_{i j}$ is the Euclidean distance between the two spots. We calculate CHAOS as the mean length of the graph edges in the $1 \mathrm{NN}$ graph in the following form

$$
\text { CHAOS }=\frac{\sum_{k=1}^{K} \sum_{i, j}^{n_{k}} w_{k i j}}{N}
$$

where $N$ is the total number of spots; $K$ is the total number of spatial domains; and $n_{k}$ is the number of spots in $k$-th spatial domain. A smaller CHAOS score reflects better spatial continuity. 
The PAS score measures the randomness of the spots that are located outside of the spatial domain where it was clustered to. The PAS score is calculated as the proportion of spots with a cluster label that is different from at least 6 of its neighboring 10 spots. A small PAS score indicates spot homogeneity within spatial clusters.

\section{Cell type deconvolution.}

We performed deconvolution using the software RCTD with default settings ${ }^{134}$. Cell type deconvolution in spatial transcriptomics allows us to estimate the cell type composition on each spatial location using a reference scRNA-seq data.

For the DLPFC data, we used the same scRNA-seq data that we used as reference in simulations 102 for deconvolution. The scRNA-seq reference data contains expression measurements for 24,153 genes and 2,394 cells that belong to six cell types annotated in the original study. The six cell types include astrocytes $(n=76$ cells), GABAergic neurons $(n=701$ cells), microglia $(n=68)$, neurons $(n=1057)$, oligodendrocyte progenitor cell (OPC; $n=117)$, and stem cells $(n=290)$.

For the Slide-seq data, we selected a scRNA-seq data collected on the same tissue, the mouse cerebellum, via Drop-seq ${ }^{135}$ to serve as the reference data for deconvolution. The reference data contains 26,139 cells that belong to multiple cell types characterized in the original study. We focus our analysis on major cell types that include granule cell ( $\mathrm{n}=21,331$ cells), Purkinje neuron $(n=178)$, interneuron $(n=1,547)$, oligodendrocyte $(n=347)$, astrocyte $(n=326)$, Bergmann glia $(n=1,404)$, microglia $(n=64)$, choroid plexus $(n=50)$, endothelial cell $(n=422)$, fibroblast-like cell $(\mathrm{n}=241)$, and interneurons_and_Other_Nnat $(\mathrm{n}=229)$.

For the Slide-seq V2 data, we selected a scRNA-seq data collected on the same tissue, the mouse hippocampus, via Drop-seq ${ }^{135}$, to serve as the reference data for deconvolution. The reference data contains 53,204 cells that belong to multiple cell types characterized in the original study. The cell types include Anterior Subiculum, proximal to CA1 $(n=1,556)$, Astrocyte $(n=7,503)$, CA1 Principal cells $(n=2,489)$, CA1 Principal cells (Anterior) $(n=3,088)$, CA2 Principal cells $(n=330)$, CA3 Principal cells $(n=5,623)$, Cajal-retzius $(n=336)$, Choroid_Plexus $(n=22)$, Deep layer subiculum ( $n=509)$, Dentate hilum $(n=530)$, Dentate Principal cells $(n=13,265)$, Endothelial_Stalk $(n=1,903)$, Endothelial_Tip $(n=296)$, Entorhinal cortex $(n=1,580)$, Entorhinal cortex (IEG) $(n=27)$, Ependymal $(n=337)$, Interneuron $(n=3,035)$, Lateral CA3 Principal cells $(n=649)$, Medial entorrhinal cortex $(n=867)$, Medial entorrhinal cortexm $(n=56)$, Microglia $(n=467)$, Mural $(n=705)$, MyelinProcesses $(n=227)$, Neurogenesis (SGZ) $(n=458)$, Neuron $(n=3,087)$, Oligodendrocyte $(n=1,969)$, Polydendrocyte $(n=1,046)$, Postsubiculum $(n=43)$, Resident macrophage $(n=78)$, Subiculum $(\mathrm{n}=1,123)$.

1001

1002

1003

For the ST data, we selected two scRNA-seq data collected on breast cancer to serve as the reference data for deconvolution. Because the composition and density of immune cells in the tumor microenvironment can influence tumor growth, cancer progression, we collected a reference data with multiple immune cells. The first reference data is collected on breast cancer with dropletbased scRNA-Seq performed on the single-cell suspensions using the Chromium Single Cell 3' v2

1004 Library in 10X Genomics ${ }^{63}$. It contains a larger sample size with 47,016 cells that belong to 1005 multiple immune cell types characterized in the original study. These major immune cell types 
1006

1007

1008

1009

1010

1011

1012

1013

1014

1015

1016

1017

1018

1019

1020

1021

1022

1023

1024

1025

1026

1027

1028

1029

1030

1031

1032

1033

1034

1035

1036

1037

1038

1039

1040

1041

1042

1043

1044

1045

1046

1047

1048

1049

1050

1051

include monocytes $(n=816$ cells), macrophages $(n=2,104)$, myeloid dendritic cells (mDCs; $\mathrm{n}=1,345)$, plasmacytoid dendritic cells ( $\mathrm{pDCs} ; \mathrm{n}=166)$, T CD4 cells $(\mathrm{n}=17,183)$, T CD8 cells $(n=10,987)$, T Reg cells $(n=309)$, B cells $(n=3,889)$, mast cells $(n=558)$, and neutrophils $(n=439)$. The second reference data is collected on breast cancer via the InDrop platform ${ }^{136}$. It contains 24,271 cells that belong to multiple immune cell types and malignant cell types characterized in the original study. These cell types include B_Cells $(n=1,245)$, CD4+ T-cells $(n=2,003)$, CD8+ Tcells $(n=3,691)$, Myeloid ( $n=4,606)$, Myoepithelial $(n=212)$, NK cells $(n=358)$, NKT cells $(n=164)$, Plasma_Cells $(n=1,955)$, T_cells_unassigned $(n=938)$, T-cells Cycling (proliferating T cells, $\mathrm{n}=605)$, T-Regs $(\mathrm{n}=994)$, Tfh cells (T-follicular helper, $\mathrm{n}=175)$, dPVL (differentiatedperivascular-like, $n=214$ ), iCAFs (inflammatory cancer-associated fibroblast, $n=1,129$ ), imPVL (immature-PVL, $\mathrm{n}=106$ ), myCAFs (myofibroblast-like CAF, $\mathrm{n}=280$ ), Endothelial $(\mathrm{n}=610$ ), Epithelial_Basal (cancer cells, n=4,095), Epithelial_Basal_Cycling (cancer cells with high proliferation, $n=614$ ), and Epithelial_Luminal_Mature (epithelial normal luminal, n=277). We provide the deconvolution analysis results with the first reference data in the main text and provided the results based on the second reference data in the supplementary figures.

For each dataset, with the scRNA-seq reference data, we estimated the cell type composition of the reference cell types on each spatial location.

\section{High-resolution spatial map construction and gene expression prediction.}

We constructed high-resolution spatial map for the ST tumor data with relatively low resolution. To do so, we scaled the $\mathrm{x}$ - and $\mathrm{y}$-coordinates of the measured spatial locations to have mean zero and unit standard deviation. We then replaced each measured spatial location with four new locations by adding or subtracting the same small distance on both $\mathrm{x}$ and $\mathrm{y}$ coordinates of the measured location. Subsequently, the four new locations form a square, with the original measured location sitting in the center. The small distance used to create the new locations is data dependent: it is calculated as $1 / 4$ of the median distance between one location with its nearest neighbor. This small distance is calculated to be 0.035 in the ST tumor data. We imputed spatial PCs and the expression level of individual genes on the new locations.

\section{Trajectory inference on the tissue.}

Besides spatial domain detection, we also applied Slingshot ${ }^{20}$ on the low-dimensional components to infer the developmental trajectories among spatial locations on the tissue in the DLPFC, ST tumor, and Slideseq V2 data. We performed trajectory inference on all locations for the first two datasets. For the Slideseq V2 data, we focused trajectory inference only on the three cortical layers because slingshot cannot handle the large sample size on the whole tissue slice and because only the cortical layers are naturally ordered as they are developed in a sequential fashion during cortico-genesis. Slingshot is a common scRNA-seq trajectory inference method originally applied on regular PCs. Here, we applied slingshot on the spatial PCs so that the nearby tissue locations with similar gene expression will be inferred with similar pseudo-time. Slingshot requires the cluster labels as additional input. We used the cluster labels obtained from the clustering analysis described in a previous subsection for Slingshot. After trajectory inference, Slingshot assigned each location a pseudo-time value. Slingshot requires users to specify the start of the trajectory (i.e. beginning of pseudo-time) based on biological knowledge. In the trajectory analysis, almost all lineage inference methods ${ }^{22 ; 31 \text {; }}$ require the user to designate one cluster as the start cluster, or one cell as the start cell. Setting a start cluster only changes the direction of the trajectory but does not 
1052 influence the relative position of clusters on the trajectory. We set the white matter as the start 1053 cluster for DLPFC, set the tumor region as the start cluster for ST, and set the layer 6 as start cluster 1054 for Slide-seq V2. In Slide-seq V2 data, we focused the trajectory inference only on the cortical layers 4, 5 and 6, because slingshot cannot handle the large sample size on the whole tissue slice and because the cortical layers are naturally ordered as they are developed in a sequential fashion during cortico-genesis. In ST data, we focused on trajectory inference on tumor and tumor adjacent regions to investigate how these locations are connected to one another and underlie tumorigenesis. Based on the inferred pseudo-time values, we connected neighboring locations on the tissue to construct trajectories. Specifically, we first overlayed the tissue in each data with an evenly spaced square grid graph. For each smallest square on the grip graph, we obtained inside the square the two locations that have the largest and smallest pseudo-time values. We then draw an arrow line connecting these two locations, with the arrow pointing towards the location with the larger pseudo-time. The size of the grid graph is data dependent: we searched in sequence a set of predetermined numbers $(10,15,20,25,30)$ as grid size choice for each dataset, and selected among

1066 them the smallest number that ensures at least $1 / 3$ of the arrow lines to start from one spatial domain and end at another spatial domain. This way, an appreciable fraction of arrow lines would cross regional boundaries, ensuring effective visualization of trajectories between spatial domains. With the strategy, we used a 10 by 10 grid for the ST data and a 20 by 20 grid for the DLPFC data. We also applied stLearn on DLPFC sample 151673 to infer the trajectory and we set the spot on the left-bottom corner of white matter as the root spot in the inference. We did not apply stLearn to the other datasets due to a lack of H\&E image in Slide-seq and Slide-seq V2 data, and a lack of JSON file in the HER2-positive breast tumor data.

\section{Differential gene expression analysis and enrichment analysis}

We performed differential gene expression (DE) analyses and subsequent gene set enrichment analyses on all of the four datasets. We performed two sets of DE analyses. The first set of DE analyses aims to identify spatial domain specific DE genes. In particular, we examined the SpatialPCA detected spatial domains one at a time and used MAST ${ }^{137}$ wrapped in the FindMarkers function in the Seurat package to identify genes that are DE in the domain of focus as compared to all other domains at a false discovery rate (FDR) of 0.05 . The second set of DE analyses aims to identify pseudo-time associated genes. In particular, we used Pearson's correlation test to identify genes that were associated with the inferred pseudo-time based on a Bonferroni corrected $p$-value threshold of 0.05 . After each set of DE analyses, we performed gene set enrichment analyses (GSEA) on the detected DE genes using the g:GOSt function in gProfiler2 package. In the enrichment analysis, we used all expressed genes as background ${ }^{138}$ and used the default option $\mathrm{g}$ :SCS method in gProfiler2 for multiple testing correction. The gene sets are downloaded from the Molecular Signatures Database $\left(\mathrm{MSigDB}^{139 ; 140}\right)$ available from the Broad Institute, including the $\mathrm{C} 2$ (KEGG), C5 (GO BP: biological processes, GO CC: cellular components, GO MF: molecular functions and HP :Human Phenotype Ontology) and hallmark pathway datasets. For the ST tumor data, we also included cancer related gene sets including C4 (cancer modules) and C7

1092 (immunologic signatures). For the Slide-seq cerebellum data, we converted the MGI gene IDs to 1093 human homolog gene symbols before GSEA analysis ${ }^{141}$. 


\section{Availability of data and materials}

1095

1096

1097

1098

1099

1100

1101

1102

1103

1104

1105

1106

1107

\section{Funding}

1109

1110

1111

1112 ID SCP815.
The SpatialPCA software code are publicly available at http://xzlab.org/software.html. The source code is released under the GNU General Public License version 3 (GPL >=3). All analysis code for reproducing the results of the present study are publicly available at http://lulushang.org/docs/Projects/SpatialPCA. The human DLPFC samples are available at http://spatial.libd.org/spatialLIBD/. ST data is available at https:/github.com/almaan/her2st. Slide-seq data is available at Broad Institute's single-cell repository (https://singlecell.broadinstitute.org/single_cell/) with ID SCP354. Slide-seq V2 data is available at Broad Institute's single-cell repository (https://singlecell.broadinstitute.org/single_cell/) with

This study was supported by the National Institutes of Health (NIH) Grants R01GM126553, R01HG011883, and R01GM144960. 
bioRxiv preprint doi: https://doi.org/10.1101/2022.01.19.476966; this version posted January 21, 2022. The copyright holder for this preprint (which was not certified by peer review) is the author/funder. All rights reserved. No reuse allowed without permission.

\section{Figure 1}

a.
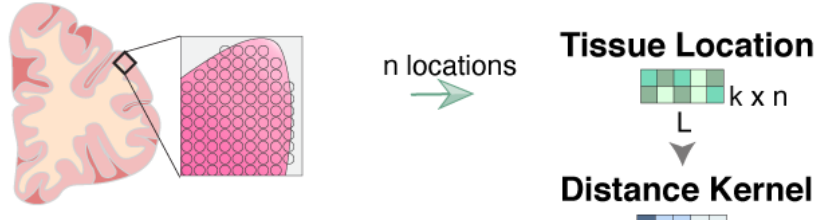

\section{Distance Kernel}

\section{SpatialPCA}

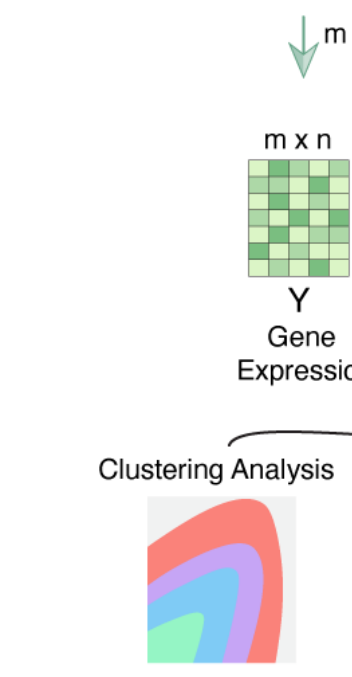

Spatial Domain Detection

High-resolution Spatial Map Construction

Developmental Trajectory Analysis

b.
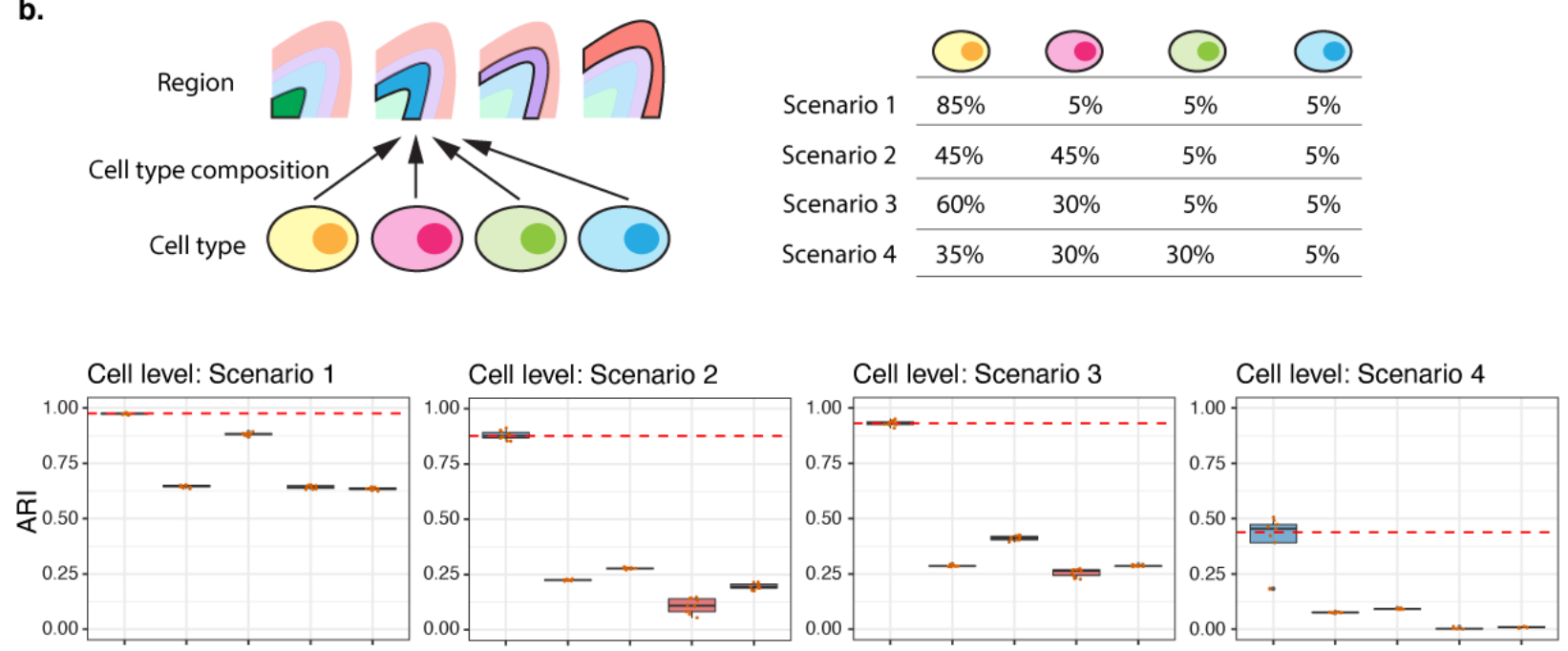

1114
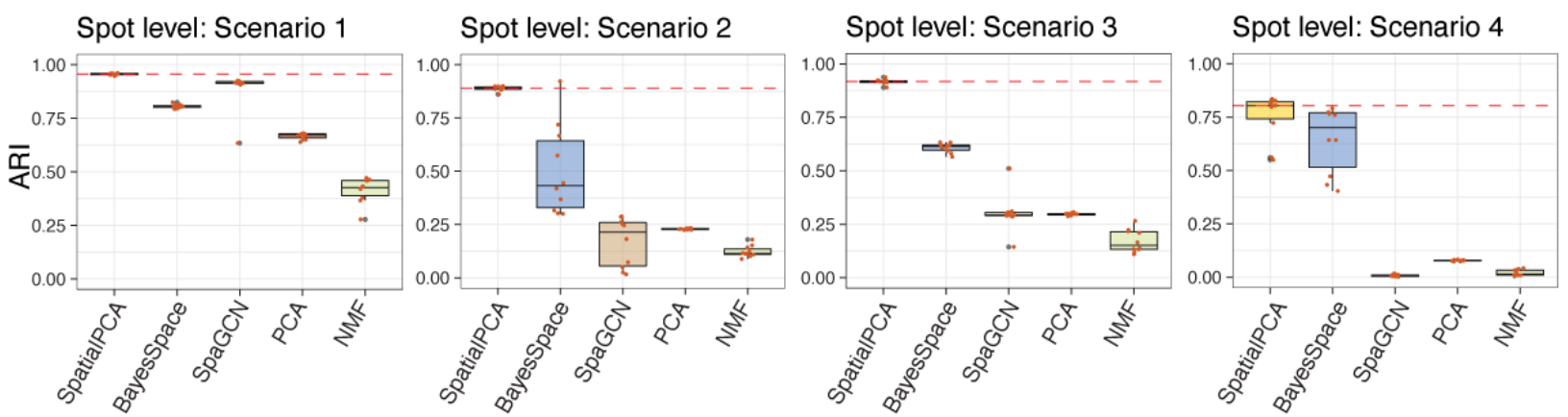

$1 \mid 115$ 
1117 Figure 1. Method schematic of SpatialPCA and simulation results. (a). SpatialPCA is a 1118 spatially aware dimension reduction method that takes both gene expression $\boldsymbol{Y}$ and location information $\boldsymbol{L}$ as input. It models the gene expression matrix $\boldsymbol{Y}$ as a function of latent factors $\boldsymbol{Z}$ through a factor analysis model. Importantly, SpatialPCA builds a kernel matrix $\boldsymbol{\Sigma}$ using the location information $\boldsymbol{L}$ to explicitly model the spatial correlation structure in the latent factors $\boldsymbol{Z}$ across tissue locations. Consequently, the inferred low dimensional components $\boldsymbol{Z}$ from SpatialPCA contain valuable spatial correlation information and can be paired with various analytic tools already developed in scRNA-seq studies to enable effective and novel downstream analyses for spatial transcriptomics. The examined downstream analyses of spatial transcriptomics include spatial transcriptomics visualization, spatial domain detection, trajectory inference on the tissue, and high-resolution spatial map construction. (b). In simulation, we obtained cortex tissue from the DLPFC data and manually segmented it into four cortical layers. We specified a distinct cell type composition for each cortical layer and simulate four scenarios. Then we assigned the simulated cells onto the locations of the cortex based on the specified cell type composition in each layer to create the spatial transcriptomics data. We simulated spatial transcriptomics data both at single-cell resolution and spot-level data. We use adjusted Rand index (ARI) to measure the spatial clustering accuracy at single cell resolution and spot level (spot diameter is 90um). The higher ARI indicates better spatial clustering performance. SpatialPCA outperforms the other methods for detecting the spatial domains in the simulations. 
Figure 2

a.
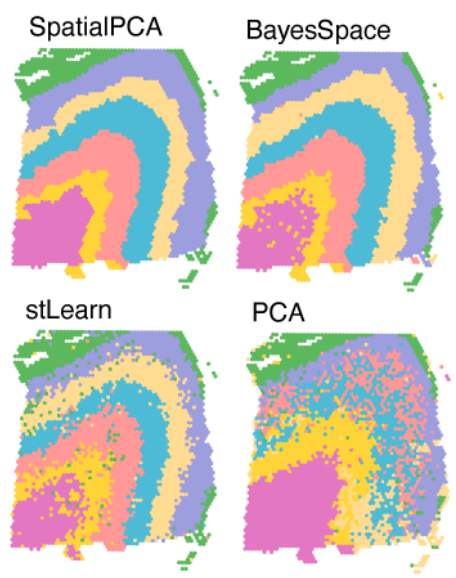

c. SpatialPCA RGB
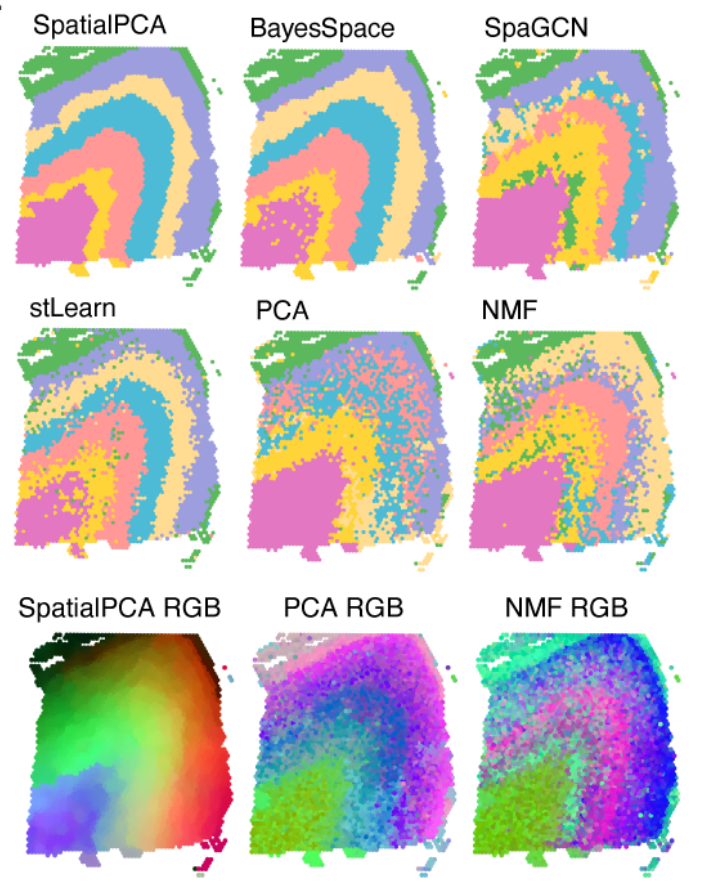

f.

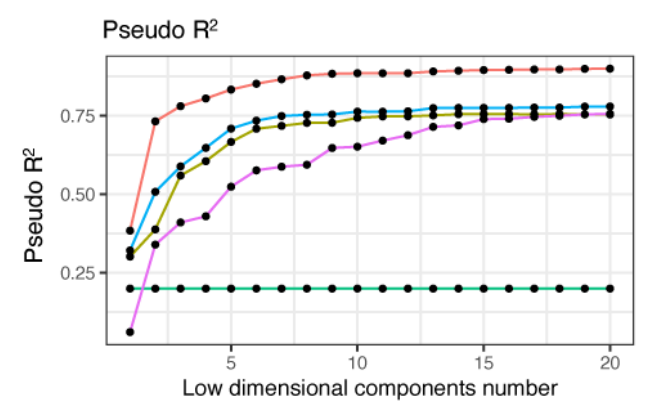

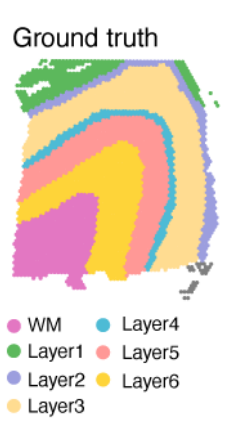

b.

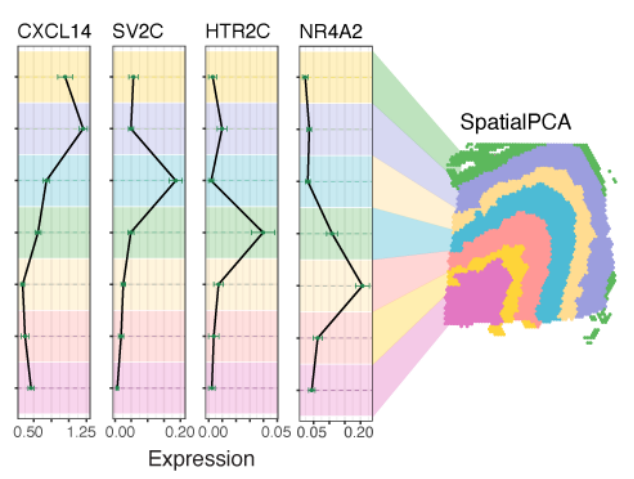

d.

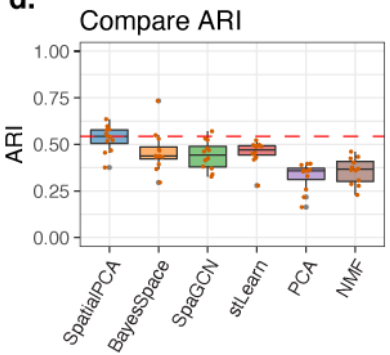

e. Compare LISI
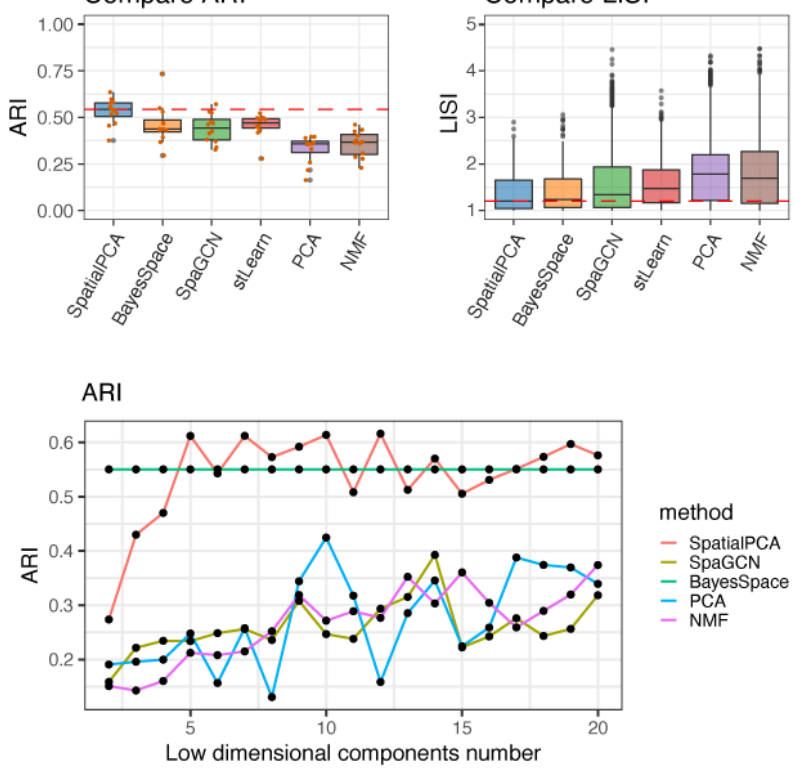

Figure 2. Analysis of the cortex data from DLPFC. (a) Clustering of tissue locations based on SpatialPCA, BayesSpace, SpaGCN, stLearn, PCA and NMF. Ground truth of tissue regions of the human prefrontal cortex are annotated by the original DLPFC study. (b) Mean expression of layer specific markers including layer 2 marker gene $C X C L 14$, layer 3 marker gene $S V 2 C$, layer 5 marker gene $H T R 2 C$, and layer 6/6b marker gene $N R 4 A 2$. The cluster labels are corresponded to the labels of SpatialPCA detected spatial domains in figure (a). (c) For SpatialPCA, PCA, and NMF, we summarized the inferred low dimensional components into three UMAP components and visualized the three resulting components with red/green/blue (RGB) colors through the RGB plot. (d) Clustering accuracy of different methods in recapitulating the true tissue domains. Accuracy is measured by ARI. For BayesSpace and SpaGCN, clustering was performed based on their default settings. For dimension reduction methods (PCA and NMF), clustering was performed based on the inferred low-dimensional components on spatially variable genes. (e) Clustering performance of different methods in obtaining smooth and continuous spatial domains measured by local inverse Simpson's index (LISI). Lower LISI score indicates more homogeneous neighborhood spatial domain clusters of a spot. (f) Spatial PCs in SpatialPCA have higher prediction accuracy for the ground truth of tissue regions in terms of McFadden's pseudo- $\mathrm{R}^{2}$ (left) and ARI (right) than 
bioRxiv preprint doi: https://doi.org/10.1101/2022.01.19.476966; this version posted January 21, 2022. The copyright holder for this preprint (which was not certified by peer review) is the author/funder. All rights reserved. No reuse allowed without permission.

1156 latent components from PCA, NMF and SpaGCN. For BayesSpace, we treated its inferred cluster 1157 labels as the predictors. 
bioRxiv preprint doi: https://doi.org/10.1101/2022.01.19.476966; this version posted January 21, 2022. The copyright holder for this preprint (which was not certified by peer review) is the author/funder. All rights reserved. No reuse allowed without permission.

1158 Figure 3

a. Granule cell layer

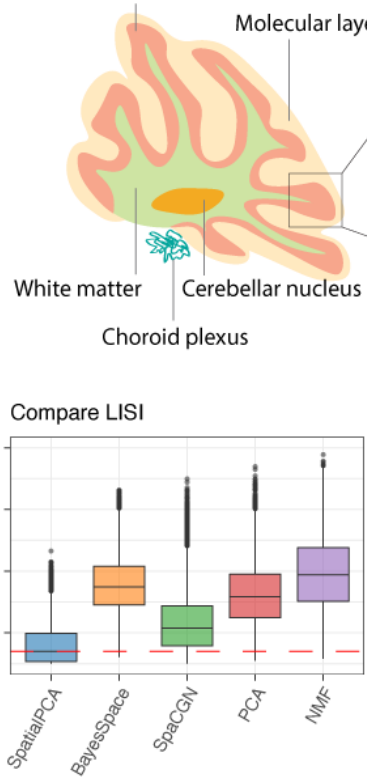

f. BayesSpace

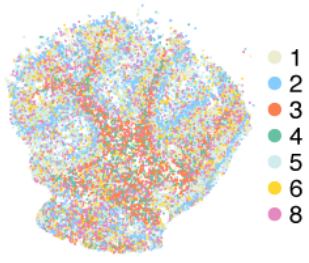

SpaGCN

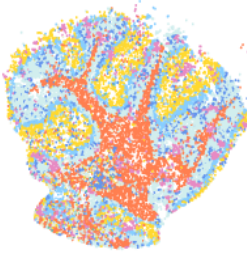

1
-2
-3
-4
5
-6
6
7
8
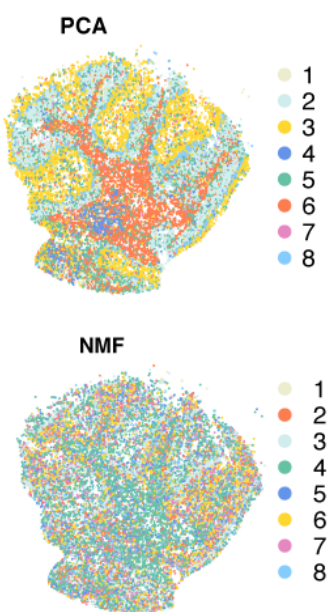

b.

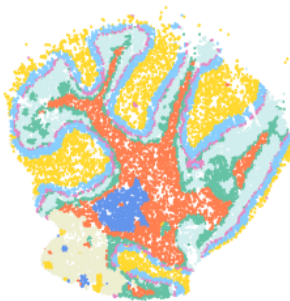

SpatialPCA

Molecular layer

Purkinje layer

Granule outer sublayer

Granule middle sublayer

Granule inner sublayer

White matter

- Cerebellar nucleus

Choroid plexus

\section{e.}

d. Cst3

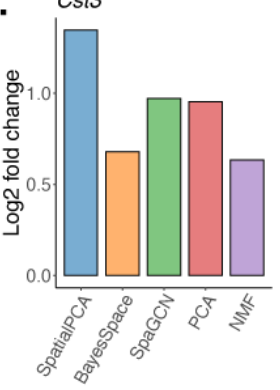

Molecular layer 0

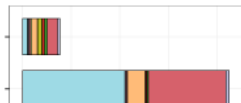

CellType

Purkinje layer

Granule outer sublayer

Granule middle sublayer

Granule inner sublayer

White matter

Cerebellar nucleus

Choroid plexus

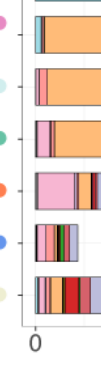

Astrocytes

Cerebellum baske

Choroid Plexus

Endothelial_Stalk

Endothelial_Tip

Fibroblast_Like

Granule_cells

Interneurons

Interneurons_and_Other_Nnat Microglia_Macrophage

Mural

Oligodendrocyte

Oligodendrocyte_Polydendrocyte

Polydendrocyte

Purkinje_Neurons

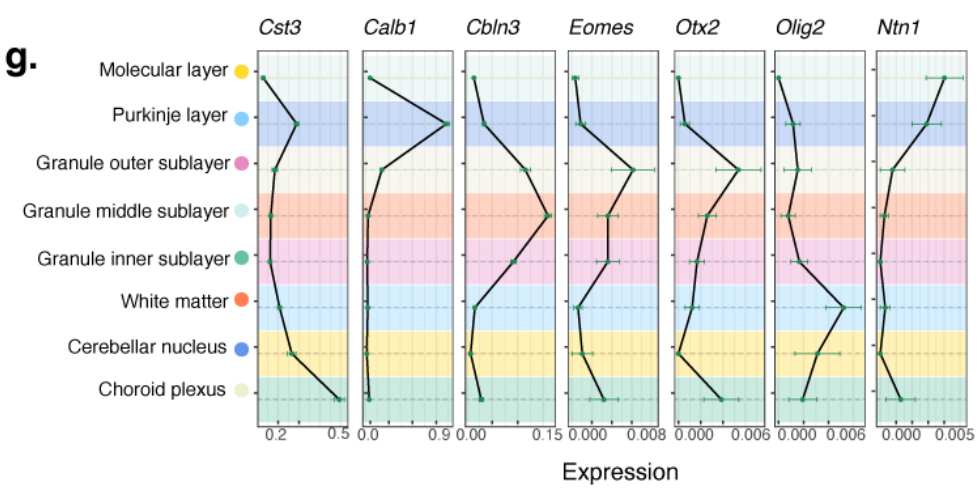

h. SpatialPCA Bergmann_Glia
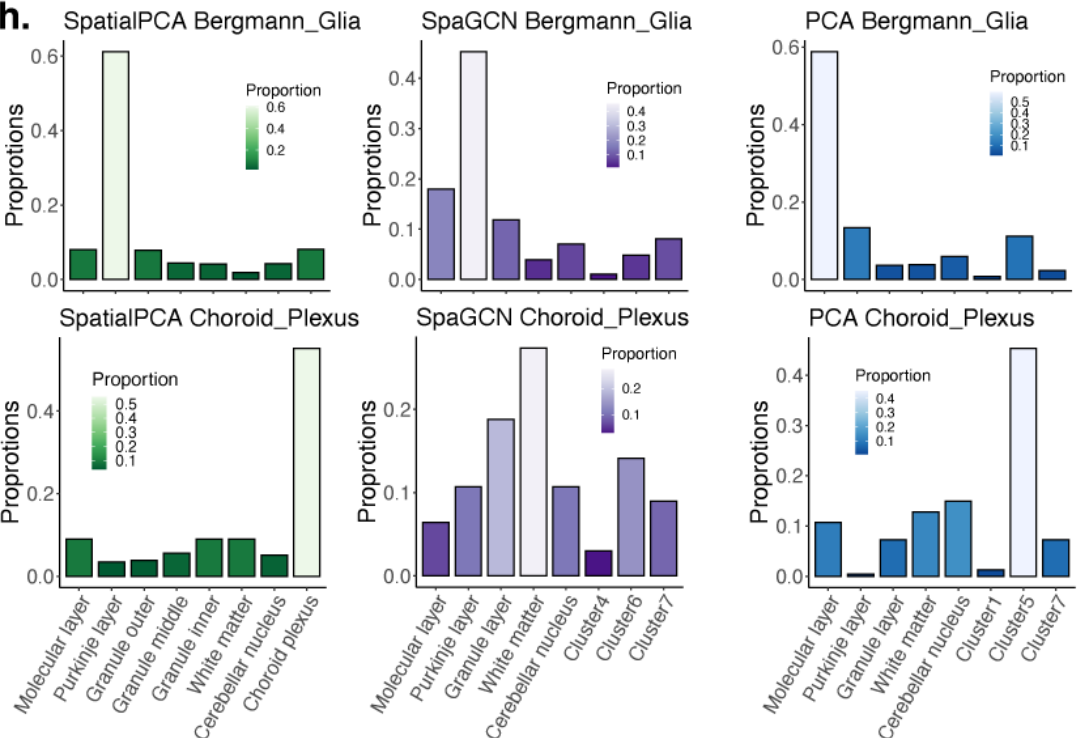

Figure 3. Analysis of the cerebellum data from Slide-seq. (a) The structure of the mouse 
1162 components inferred by SpatialPCA segregates tissue locations into distinct tissue regions. The 1163 detected tissue regions were annotated based on their relative positions on the tissue and the 1164 enriched cell types in each detected tissue domain. (c) Clustering performance of different methods 1165 in obtaining smooth and continuous spatial domains measured by local inverse Simpson's index 1166 (LISI). Lower LISI score indicates more homogeneous neighborhood spatial domain clusters of a 1167 spot. (d) The log2 fold change in mean gene expression of Cst3 gene for the cluster with highest 1168 expression compare with all other clusters in different methods. The Cst3 has highest $\log 2$ fold 1169 change in choroid plexus region in SpatialPCA. (e) Percentage of different cell types (x-axis) in 1170 each tissue domain detected by SpatialPCA (y-axis). (f) Clustering of tissue locations based on the 1171 other methods. For BayesSpace and SpaGCN, clusters were inferred directly by the software. For 1172 dimension reduction methods (PCA and NMF), clustering was performed based on the inferred 1173 low-dimensional components on spatially variable genes. (g) Mean expression of regional marker 1174 genes in cerebellum. The cluster labels are corresponded to the labels of SpatialPCA regions in 1175 figure (b). (h) Distribution of Bergmann glia cells and choroid plexus cells in each cluster for 1176 different methods. The summation of the cell type percentages in all clusters is $100 \%$ for each 1177 method. 
Figure 4

a.

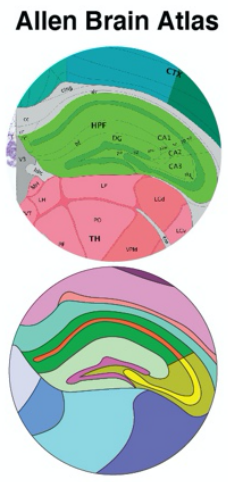

c.

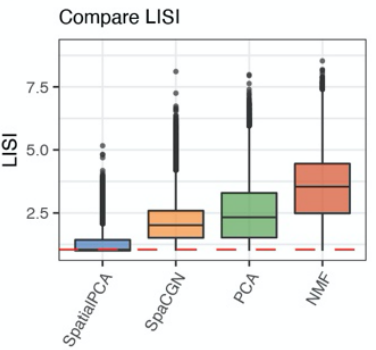

f.
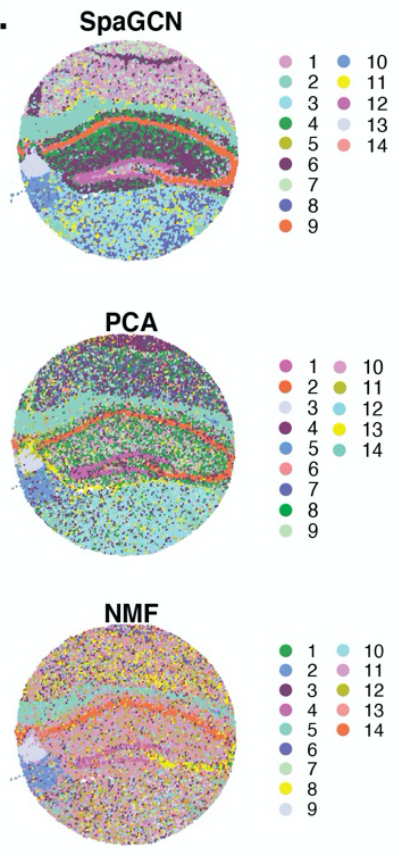

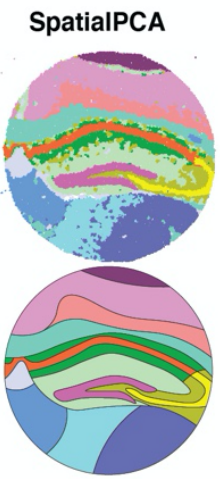

d.

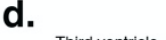

b.
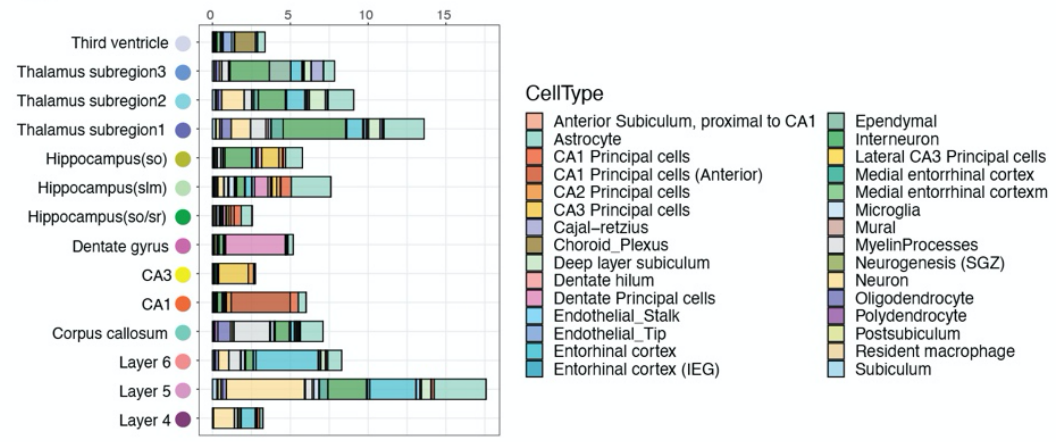

D
Myl4 Opalin Wfs 1
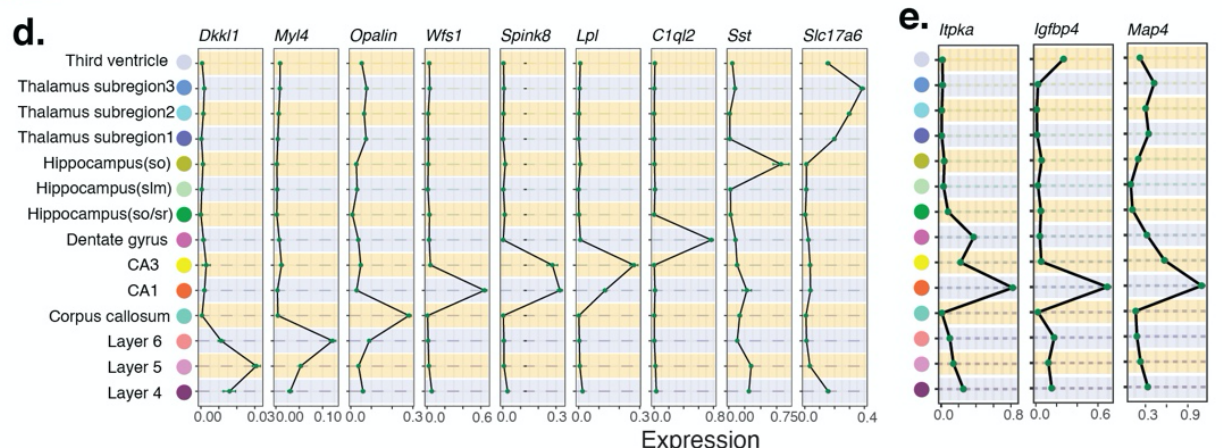

\section{g.}
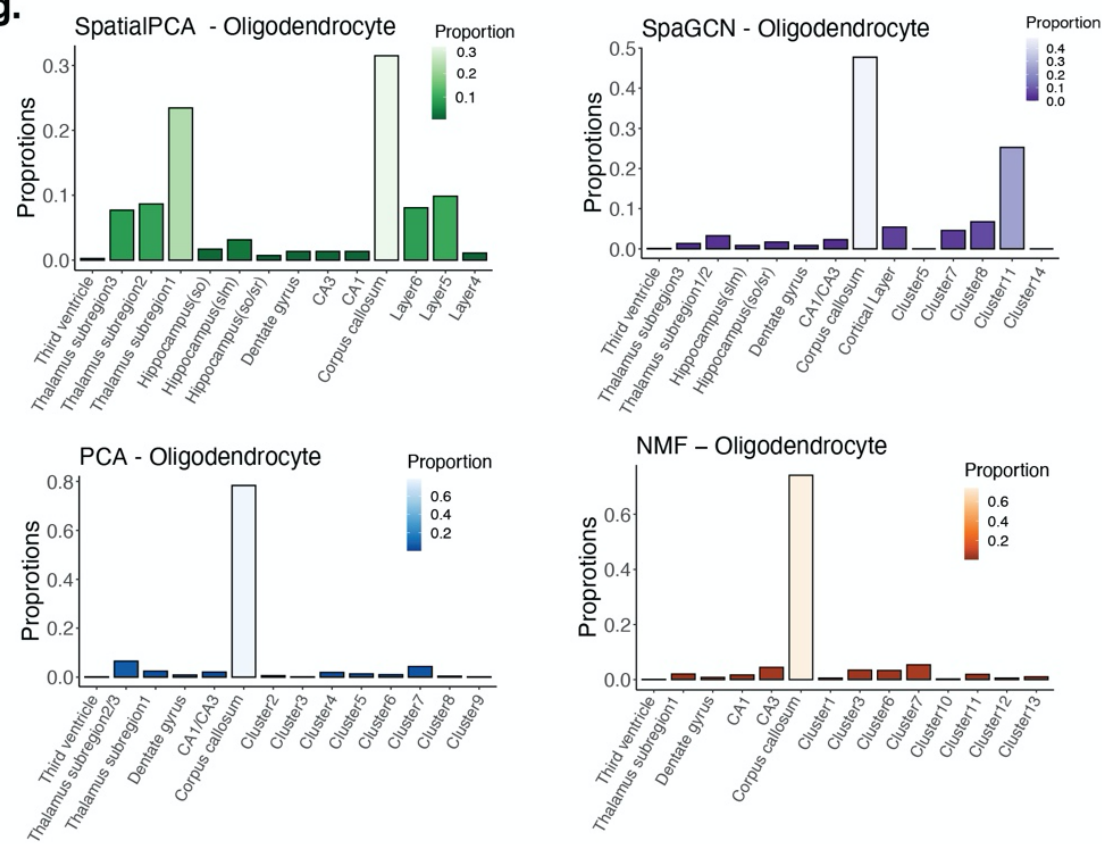

Figure 4. Analysis of the hippocampus data from Slide-seq V2. (a) Left panel: The structure of the mouse hippocampus, with the main tissue regions annotated from Allen Brain Atlas. Right panel: SpatialPCA segregates tissue locations into distinct tissue regions. Lower panel: Tissue structures based on annotations from Allen Brain Atlas and spatial domains detected by SpatialPCA. The detected tissue regions were annotated based on their relative positions on the tissue and the enriched cell types in each detected tissue domain. (b) Percentage of different cell 
1188 of different methods in obtaining smooth and continuous spatial domains measured by local 1189 inverse Simpson's index (LISI). Lower LISI score indicates more homogeneous neighborhood 1190 spatial domain clusters of a spot. (d) Mean expression of marker genes in hippocampus. (e) Mean 1191 expression of three differentially expressed genes in CA1 region that only detected by SpatialPCA 1192 clusters. (f) Clustering of tissue locations based on the other methods. For SpaGCN, clusters were 1193 inferred directly by the software. For dimension reduction methods (PCA and NMF), clustering 1194 was performed based on the inferred low-dimensional components on spatially variable genes. (g) 1195 Distribution of oligodendrocyte cells in each cluster for different methods. The summation of the 1196 cell type percentages in all clusters is $100 \%$ for each method.

1197

1198 
Figure 5

a.

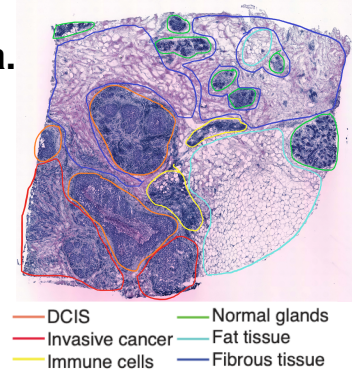

c. ARI

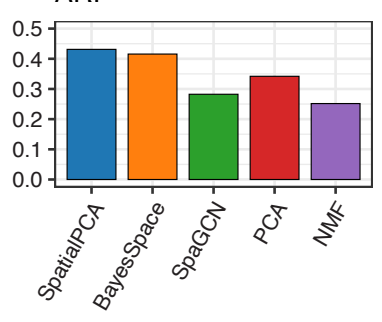

e.

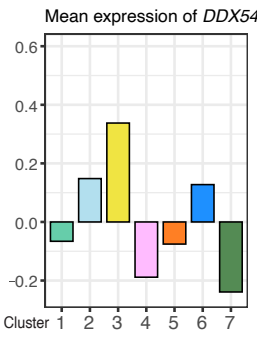

g.

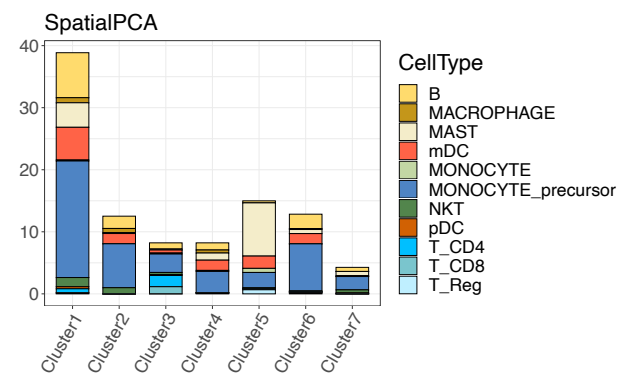

b.

d.

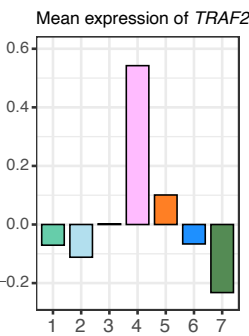

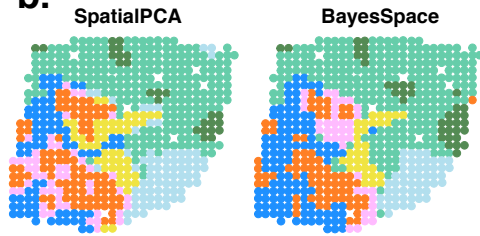
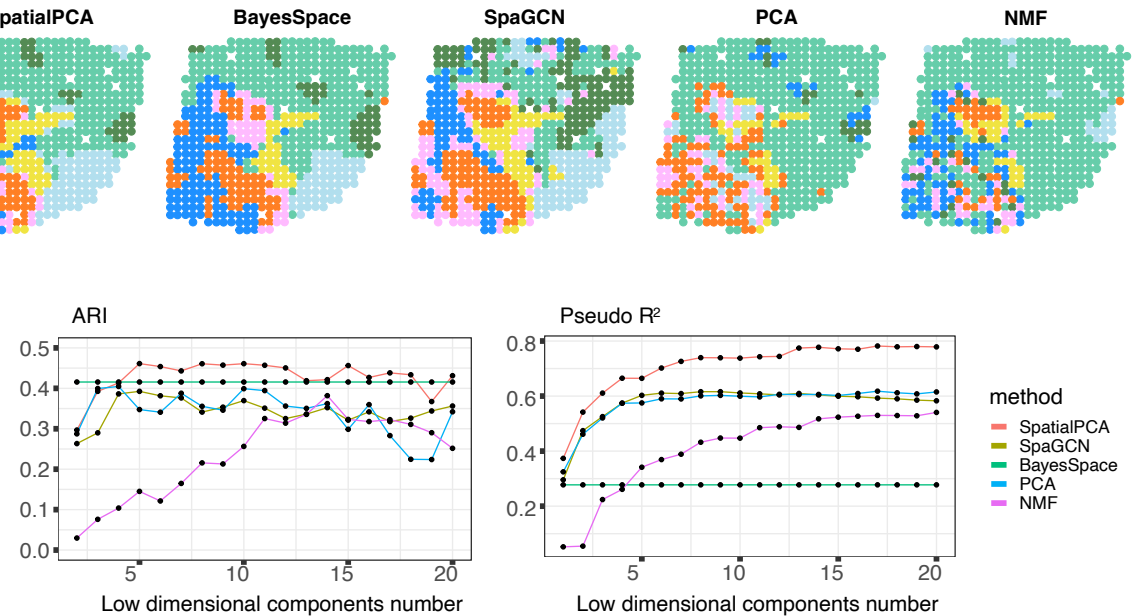

f.
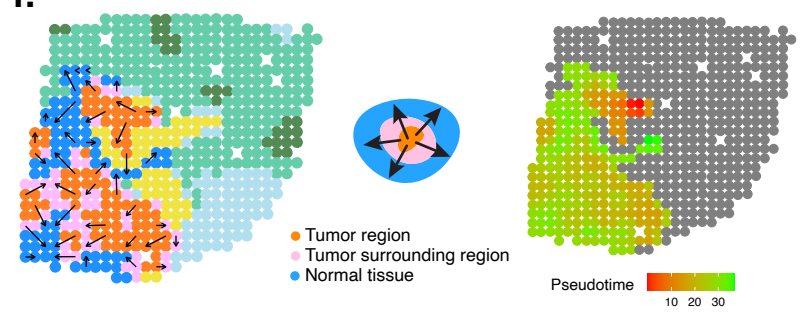

h.

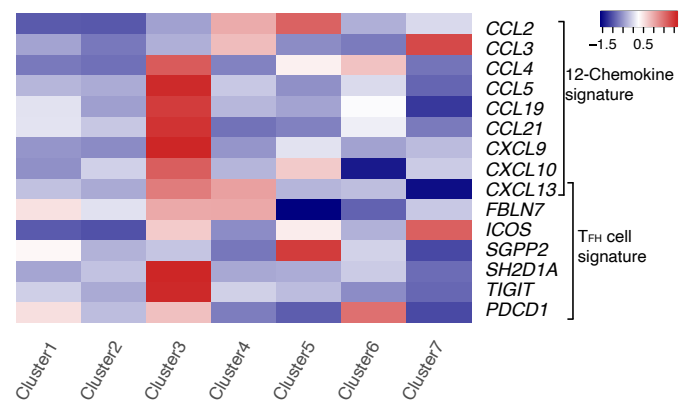

Clusters: 1- Fibrous tissue near normal glands 2 - Tumor surrounding region 3 - Immune region 4 - Tumor region 5 - Fibrous tissue near tumor 6 - Normal glands 7 - Fat tissue

Figure 5. Analysis of the HER2 tumor data from ST. (a) Haematoxylin and eosin (H\&E) staining image shows distinct tissue regions annotated by a pathologist in the original study. The annotated tissue regions include invasive cancer (red), fat tissue (cyan), fibrous tissue (blue), normal breast glands (green), in situ cancer/DCIS (orange), and immune cells (yellow). (b) Clustering of tissue locations using different methods. For BayesSpace and SpaGCN, clusters were inferred directly by the software. For dimension reduction methods (SpatialPCA, PCA and NMF), clustering was performed based on the inferred low-dimensional components on spatially variable genes. (c) Clustering accuracy of different methods in recapitulating the true tissue regions. Accuracy is measured by ARI. (d) Spatial PCs in SpatialPCA have higher prediction accuracy in terms of ARI (left) and McFadden's pseudo- $\mathrm{R}^{2}$ (right) for the ground truth labels than latent components in PCA, NMF and SpaGCN. For BayesSpace, we treated its inferred cluster labels as 
1214 the predictors. (e) Mean expression of immune region differentially expressed gene $D D X 54$ and 1215 tumor surrounding region differentially expressed gene $T R A F 2$ that only detected through 1216 SpatialPCA clusters. Figure legend on the bottom relates the cluster numbers detected by 1217 SpatialPCA to tissue domain names. (f) Left: visualization of the trajectory inferred by SpatialPCA. 1218 Middle: diagram shows the directionality of the trajectory, which points from the tumor region 1219 towards the normal tissue through the tumor surrounding region. Right: Arrows point from tissue 1220 locations with low pseudo-time to tissue locations with high pseudo-time. (g) The percentage of 1221 cell types inferred through cell type deconvolution analysis on each tissue domain detected by 1222 SpatialPCA. (h) Heatmap shows the mean expression level of multiple tertiary lymphoid structure 1223 (TLS) signature genes on different tissue domains detected by SpatialPCA. 


\section{References}

1228

1229

1230

1231

1232

1233

1234

1235

1236

1237

1238

1239

1240

1241

1242

1243

1244

1245

1246

1247

1248

1249

1250

1251

1252

1253

1254

1255

1256

1257

1258

1259

1260

1261

1262

1263

1264

1265

1266

1267

1268

1. Stickels, R.R., Murray, E., Kumar, P., Li, J., Marshall, J.L., Di Bella, D., Arlotta, P., Macosko, E.Z., and Chen, F. (2020). Sensitive spatial genome wide expression profiling at cellular resolution. bioRxiv.

2. Rodriques, S.G., Stickels, R.R., Goeva, A., Martin, C.A., Murray, E., Vanderburg, C.R., Welch, J., Chen, L.L.M., Chen, F., and Macosko, E.Z. (2019). Slide-seq: A scalable technology for measuring genome-wide expression at high spatial resolution. Science 363, 1463-+.

3. Wang, X., Allen, W.E., Wright, M.A., Sylwestrak, E.L., Samusik, N., Vesuna, S., Evans, K., Liu, C., Ramakrishnan, C., Liu, J., et al. (2018). Three-dimensional intact-tissue sequencing of single-cell transcriptional states. Science 361.

4. Stahl, P.L., Salmen, F., Vickovic, S., Lundmark, A., Navarro, J.F., Magnusson, J., Giacomello, S., Asp, M., Westholm, J.O., Huss, M., et al. (2016). Visualization and analysis of gene expression in tissue sections by spatial transcriptomics. Science 353, 78-82.

5. 10x Genomics: Visium Spatial Gene Expression. 10x Genomics, https://www.10xgenomics.com/solutions/spatial-gene-expression/.

6. Ke, R., Mignardi, M., Pacureanu, A., Svedlund, J., Botling, J., Wahlby, C., and Nilsson, M. (2013). In situ sequencing for RNA analysis in preserved tissue and cells. Nat Methods $10,857-860$.

7. Lee, J.H., Daugharthy, E.R., Scheiman, J., Kalhor, R., Ferrante, T.C., Terry, R., Turczyk, B.M., Yang, J.L., Lee, H.S., Aach, J., et al. (2015). Fluorescent in situ sequencing (FISSEQ) of RNA for gene expression profiling in intact cells and tissues. Nat Protoc 10, 442-458.

8. Chen, K.H., Boettiger, A.N., Moffitt, J.R., Wang, S., and Zhuang, X. (2015). RNA imaging. Spatially resolved, highly multiplexed RNA profiling in single cells. Science 348, aaa6090.

9. Moffitt, J.R., Hao, J., Wang, G., Chen, K.H., Babcock, H.P., and Zhuang, X. (2016). Highthroughput single-cell gene-expression profiling with multiplexed error-robust fluorescence in situ hybridization. Proc Natl Acad Sci U S A 113, 11046-11051.

10. Moffitt, J.R., Hao, J., Bambah-Mukku, D., Lu, T., Dulac, C., and Zhuang, X. (2016). Highperformance multiplexed fluorescence in situ hybridization in culture and tissue with matrix imprinting and clearing. Proc Natl Acad Sci U S A 113, 14456-14461.

11. Shah, S., Lubeck, E., Zhou, W., and Cai, L. (2016). In Situ Transcription Profiling of Single Cells Reveals Spatial Organization of Cells in the Mouse Hippocampus. Neuron 92, 342-357.

12. Lubeck, E., Coskun, A.F., Zhiyentayev, T., Ahmad, M., and Cai, L. (2014). Single-cell in situ RNA profiling by sequential hybridization. Nat Methods 11, 360-361.

13. Eng, C.L., Lawson, M., Zhu, Q., Dries, R., Koulena, N., Takei, Y., Yun, J., Cronin, C., Karp, C., Yuan, G.C., et al. (2019). Transcriptome-scale super-resolved imaging in tissues by RNA seqFISH. Nature 568, 235-239.

14. Asp, M., Bergenstrahle, J., and Lundeberg, J. (2020). Spatially Resolved Transcriptomes-Next Generation Tools for Tissue Exploration. Bioessays 42, e1900221.

15. Crosetto, N., Bienko, M., and van Oudenaarden, A. (2015). Spatially resolved transcriptomics and beyond. Nat Rev Genet 16, 57-66.

16. Rao, A., Barkley, D., Franca, G.S., and Yanai, I. (2021). Exploring tissue architecture using spatial transcriptomics. Nature 596, 211-220. 
17. Sun, S., Zhu, J., Ma, Y., and Zhou, X. (2019). Accuracy, robustness and scalability of dimensionality reduction methods for single-cell RNA-seq analysis. Genome Biol 20, 269.

18. Kiselev, V.Y., Kirschner, K., Schaub, M.T., Andrews, T., Yiu, A., Chandra, T., Natarajan, K.N., Reik, W., Barahona, M., Green, A.R., et al. (2017). SC3: consensus clustering of single-cell RNA-seq data. Nat Methods 14, 483-486.

19. Saelens, W., Cannoodt, R., Todorov, H., and Saeys, Y. (2019). A comparison of single-cell trajectory inference methods. Nat Biotechnol 37, 547-554.

20. Street, K., Risso, D., Fletcher, R.B., Das, D., Ngai, J., Yosef, N., Purdom, E., and Dudoit, S. (2018). Slingshot: cell lineage and pseudotime inference for single-cell transcriptomics. BMC Genomics 19, 477.

21. Pierson, E., and Yau, C. (2015). ZIFA: Dimensionality reduction for zero-inflated single-cell gene expression analysis. Genome Biol 16, 241.

22. Durif, G., Modolo, L., Mold, J.E., Lambert-Lacroix, S., and Picard, F. (2019). Probabilistic count matrix factorization for single cell expression data analysis. Bioinformatics 35 , 4011-4019.

23. Risso, D., Perraudeau, F., Gribkova, S., Dudoit, S., and Vert, J.P. (2018). A general and flexible method for signal extraction from single-cell RNA-seq data. Nat Commun 9, 284.

24. Stuart, T., Butler, A., Hoffman, P., Hafemeister, C., Papalexi, E., Mauck, W.M., 3rd, Hao, Y., Stoeckius, M., Smibert, P., and Satija, R. (2019). Comprehensive Integration of Single-Cell Data. Cell 177, 1888-1902 e1821.

25. Bergenstrahle, J., Larsson, L., and Lundeberg, J. (2020). Seamless integration of image and molecular analysis for spatial transcriptomics workflows. BMC Genomics 21, 482.

26. Swanton, C. (2012). Intratumor Heterogeneity: Evolution through Space and Time. Cancer Res 72, 4875-4882.

27. Janiszewska, M. (2020). The microcosmos of intratumor heterogeneity: the space-time of cancer evolution. Oncogene 39, 2031-2039.

28. Scadden, D.T. (2014). Nice neighborhood: emerging concepts of the stem cell niche. Cell 157, 41-50.

29. Zhao, E., Stone, M.R., Ren, X., Guenthoer, J., Smythe, K.S., Pulliam, T., Williams, S.R., Uytingco, C.R., Taylor, S.E.B., Nghiem, P., et al. (2021). Spatial transcriptomics at subspot resolution with BayesSpace. Nat Biotechnol.

30. Hu, J., Li, X., Coleman, K., Schroeder, A., Ma, N., Irwin, D.J., Lee, E.B., Shinohara, R.T., and Li, M. (2021). SpaGCN: Integrating gene expression, spatial location and histology to identify spatial domains and spatially variable genes by graph convolutional network. Nat Methods 18, 1342-1351.

31. Duy Pham, X.T., Jun Xu, Laura F. Grice, Pui Yeng Lam, Arti Raghubar, Jana Vukovic, Marc J. Ruitenberg, Quan Nguyen. (2020). stLearn: integrating spatial location, tissue morphology and gene expression to find cell types, cell-cell interactions and spatial trajectories within undissociated tissues. biorxiv.

32. Satija, R., Farrell, J.A., Gennert, D., Schier, A.F., and Regev, A. (2015). Spatial reconstruction of single-cell gene expression data. Nat Biotechnol 33, 495-502.

33. Maynard, K.R., Collado-Torres, L., Weber, L.M., Uytingco, C., Barry, B.K., Williams, S.R., Catallini, J.L., 2nd, Tran, M.N., Besich, Z., Tippani, M., et al. (2021). Transcriptome-scale 
spatial gene expression in the human dorsolateral prefrontal cortex. Nat Neurosci 24, 425-436.

34. Zeng, H., Shen, E.H., Hohmann, J.G., Oh, S.W., Bernard, A., Royall, J.J., Glattfelder, K.J., Sunkin, S.M., Morris, J.A., Guillozet-Bongaarts, A.L., et al. (2012). Large-scale cellularresolution gene profiling in human neocortex reveals species-specific molecular signatures. Cell 149, 483-496.

35. Gilmore, E.C., and Herrup, K. (1997). Cortical development: layers of complexity. Curr Biol 7, R231-234.

36. Agirman, G., Broix, L., and Nguyen, L. (2017). Cerebral cortex development: an outside-in perspective. FEBS Lett 591, 3978-3992.

37. Larsen, D.D., and Callaway, E.M. (2006). Development of layer-specific axonal arborizations in mouse primary somatosensory cortex. Journal of Comparative Neurology 494, 398414.

38. Marques, F., Sousa, J.C., Coppola, G., Gao, F., Puga, R., Brentani, H., Geschwind, D.H., Sousa, N., Correia-Neves, M., and Palha, J.A. (2011). Transcriptome signature of the adult mouse choroid plexus. Fluids Barriers CNS 8, 10.

39. Tu, G.F., Aldred, A.R., Southwell, B.R., and Schreiber, G. (1992). Strong conservation of the expression of cystatin C gene in choroid plexus. Am J Physiol 263, R195-200.

40. El Nagar, S., Chakroun, A., Le Greneur, C., Figarella-Branger, D., Di Meglio, T., Lamonerie, T., and Billon, N. (2018). Otx2 promotes granule cell precursor proliferation and Shhdependent medulloblastoma maintenance in vivo. Oncogenesis 7, 60.

41. Pang, Z., Jian Zuo, and James I. Morgan. (2000). Cbln3, a novel member of the precerebellin family that binds specifically to Cbln1. Journal of Neuroscience 20.17, 6333-6339.

42. Sanfilippo, C., Musumeci, G., Kazakova, M., Mazzone, V., Castrogiovanni, P., Imbesi, R., and Di Rosa, M. (2020). GNG13 Is a Potential Marker of the State of Health of Alzheimer's Disease Patients' Cerebellum. J Mol Neurosci.

43. Bao, D.S., Pang, Z., Morgan, M.A., Parris, J., Rong, Y.Q., Li, L.Y., and Morgan, J.I. (2006). Cbln1 is essential for interaction-dependent secretion of Cbln3. Mol Cell Biol 26, 93279337.

44. Miura, E., lijima, T., Yuzaki, M., and Watanabe, M. (2006). Distinct expression of Cbln family mRNAs in developing and adult mouse brains. Eur J Neurosci 24, 750-760.

45. Englund, C., Kowalczyk, T., Daza, R.A.M., Dagan, A., Lau, C., Rose, M.F., and Hevner, R.F. (2006). Unipolar brush cells of the cerebellum are produced in the rhombic lip and migrate through developing white matter. J Neurosci 26, 9184-9195.

46. Dino, M.R., and Mugnaini, E. (2008). Distribution and phenotypes of unipolar brush cells in relation to the granule cell system of the rat cochlear nucleus. Neuroscience 154, 29-50.

47. Yeung, J., Ha, T.J., Swanson, D.J., and Goldowitz, D. (2016). A Novel and Multivalent Role of Pax6 in Cerebellar Development. J Neurosci 36, 9057-9069.

48. Howarth, C., Peppiatt-Wildman, C.M., and Attwell, D. (2010). The energy use associated with neural computation in the cerebellum. J Cerebr Blood F Met 30, 403-414.

49. Magistretti, P.J., and Allaman, I. (2015). A cellular perspective on brain energy metabolism and functional imaging. Neuron 86, 883-901.

50. Kirsch, L., Liscovitch, N., and Chechik, G. (2012). Localizing genes to cerebellar layers by classifying ISH images. PLoS Comput Biol 8, e1002790. 
51. Cerminara, N.L., Lang, E.J., Sillitoe, R.V., and Apps, R. (2015). Redefining the cerebellar cortex as an assembly of non-uniform Purkinje cell microcircuits. Nature Reviews Neuroscience 16, 79-93.

52. Stickels, R.R., Murray, E., Kumar, P., Li, J., Marshall, J.L., Di Bella, D.J., Arlotta, P., Macosko, E.Z., and Chen, F. (2021). Highly sensitive spatial transcriptomics at near-cellular resolution with Slide-seqV2. Nat Biotechnol 39, 313-319.

53. Choi, B., Lee, H.W., Mo, S., Kim, J.Y., Kim, H.W., Rhyu, I.J., Hong, E., Lee, Y.K., Choi, J.S., Kim, C.H., et al. (2018). Inositol 1,4,5-trisphosphate 3-kinase A overexpressed in mouse forebrain modulates synaptic transmission and mGluR-LTD of CA1 pyramidal neurons. Plos One 13, e0193859.

54. Beletskiy, A., Chesnokova, E., and Bal, N. (2021). Insulin-Like Growth Factor 2 As a Possible Neuroprotective Agent and Memory Enhancer-Its Comparative Expression, Processing and Signaling in Mammalian CNS. International Journal of Molecular Sciences 22.

55. Tokuraku, K., Okuyama, S., Matsushima, K., Ikezu, T., and Kotani, S. (2010). Distinct neuronal localization of microtubule-associated protein 4 in the mammalian brain. Neurosci Lett 484, 143-147.

56. Andersson, A., Larsson, L., Stenbeck, L., Salmen, F., Ehinger, A., Wu, S.Z., Al-Eryani, G., Roden, D., Swarbrick, A., Borg, A., et al. (2021). Spatial deconvolution of HER2-positive breast cancer delineates tumor-associated cell type interactions. Nat Commun 12, 6012.

57. Alma Andersson, L.L., Linnea Stenbeck, Fredrik Salmén, Anna Ehinger, Sunny Wu, Ghamdan Al-Eryani, Daniel Roden, Alex Swarbrick, Åke Borg, Jonas Frisén, Camilla Engblom, Joakim Lundeberg. (2020). Spatial Deconvolution of HER2-positive Breast Tumors Reveals Novel Intercellular Relationships. bioRxiv 2020.07.14.200600.

58. Newman, A.M., Liu, C.L., Green, M.R., Gentles, A.J., Feng, W., Xu, Y., Hoang, C.D., Diehn, M., and Alizadeh, A.A. (2015). Robust enumeration of cell subsets from tissue expression profiles. Nat Methods 12, 453-457.

59. Finotello, F., Mayer, C., Plattner, C., Laschober, G., Rieder, D., Hackl, H., Krogsdam, A., Loncova, Z., Posch, W., Wilflingseder, D., et al. (2019). Molecular and pharmacological modulators of the tumor immune contexture revealed by deconvolution of RNA-seq data. Genome Med 11, 34.

60. Aponte-Lopez, A., Fuentes-Panana, E.M., Cortes-Munoz, D., and Munoz-Cruz, S. (2018). Mast Cell, the Neglected Member of the Tumor Microenvironment: Role in Breast Cancer. J Immunol Res 2018, 2584243.

61. Varricchi, G., Galdiero, M.R., Loffredo, S., Marone, G., lannone, R., Marone, G., and Granata, F. (2017). Are Mast Cells MASTers in Cancer? Front Immunol 8, 424.

62. Wang, R.F. (2006). Regulatory T cells and innate immune regulation in tumor immunity. Springer Semin Immunopathol 28, 17-23.

63. Wu, S.Z., Roden, D.L., Wang, C., Holliday, H., Harvey, K., Cazet, A.S., Murphy, K.J., Pereira, B., Al-Eryani, G., Bartonicek, N., et al. (2020). Stromal cell diversity associated with immune evasion in human triple-negative breast cancer. EMBO J 39, e104063.

64. Sautes-Fridman, C., Petitprez, F., Calderaro, J., and Fridman, W.H. (2019). Tertiary lymphoid structures in the era of cancer immunotherapy. Nat Rev Cancer 19, 307-325. 
65. Helmink, B.A., Reddy, S.M., Gao, J., Zhang, S., Basar, R., Thakur, R., Yizhak, K., Sade-Feldman, M., Blando, J., Han, G., et al. (2020). B cells and tertiary lymphoid structures promote immunotherapy response. Nature 577, 549-555.

66. Dieu-Nosjean, M.C., Giraldo, N.A., Kaplon, H., Germain, C., Fridman, W.H., and SautesFridman, C. (2016). Tertiary lymphoid structures, drivers of the anti-tumor responses in human cancers. Immunol Rev 271, 260-275.

67. Sautes-Fridman, C., Lawand, M., Giraldo, N.A., Kaplon, H., Germain, C., Fridman, W.H., and Dieu-Nosjean, M.C. (2016). Tertiary Lymphoid Structures in Cancers: Prognostic Value, Regulation, and Manipulation for Therapeutic Intervention. Front Immunol 7, 407.

68. Barone, F., Gardner, D.H., Nayar, S., Steinthal, N., Buckley, C.D., and Luther, S.A. (2016). Stromal Fibroblasts in Tertiary Lymphoid Structures: A Novel Target in Chronic Inflammation. Front Immunol 7, 477.

69. Lee, H.J., Kim, J.Y., Park, I.A., Song, I.H., Yu, J.H., Ahn, J.H., and Gong, G. (2015). Prognostic Significance of Tumor-Infiltrating Lymphocytes and the Tertiary Lymphoid Structures in HER2-Positive Breast Cancer Treated With Adjuvant Trastuzumab. Am J Clin Pathol 144, 278-288.

70. Zhao, Z.J., Ren, H.Y., Yang, F., Wang, J., Wu, G.P., and Mi, X.Y. (2015). Expression, correlation, and prognostic value of TRAF2 and TRAF4 expression in malignant plural effusion cells in human breast cancer. Diagn Cytopathol 43, 897-903.

71. Carpentier, I., Declercq, W., Malinin, N.L., Wallach, D., Fiers, W., and Beyaert, R. (1998). TRAF2 plays a dual role in NF-kappaB-dependent gene activation by mediating the TNFinduced activation of p38 MAPK and IkappaB kinase pathways. FEBS Lett 425, 195-198.

72. Milek, M., Imami, K., Mukherjee, N., Bortoli, F., Zinnall, U., Hazapis, O., Trahan, C., Oeffinger, M., Heyd, F., Ohler, U., et al. (2017). DDX54 regulates transcriptome dynamics during DNA damage response. Genome Res 27, 1344-1359.

73. Janiszewska, M., Primi, M.C., and Izard, T. (2020). Cell adhesion in cancer: Beyond the migration of single cells. J Biol Chem 295, 2495-2505.

74. Harjunpaa, H., Asens, M.L., Guenther, C., and Fagerholm, S.C. (2019). Cell Adhesion Molecules and Their Roles and Regulation in the Immune and Tumor Microenvironment. Front Immunol 10.

75. Sun, S.Q., Zhu, J.Q., and Zhou, X. (2020). Statistical analysis of spatial expression patterns for spatially resolved transcriptomic studies. Nat Methods 17, 193-+.

76. Lea, A.J., Tung, J., and Zhou, X. (2015). A Flexible, Efficient Binomial Mixed Model for Identifying Differential DNA Methylation in Bisulfite Sequencing Data. Plos Genet 11, e1005650.

77. Sun, S.Q., Hood, M., Scott, L., Peng, Q.K., Mukherjee, S., Tung, J., and Zhou, X. (2017). Differential expression analysis for RNAseq using Poisson mixed models. Nucleic Acids Research 45.

78. Sun, S., Zhu, J., Mozaffari, S., Ober, C., Chen, M., and Zhou, X. (2019). Heritability estimation and differential analysis of count data with generalized linear mixed models in genomic sequencing studies. Bioinformatics 35, 487-496.

79. Kim, T.H., Zhou, X., and Chen, M. (2020). Demystifying "drop-outs" in single-cell UMI data. Genome Biol 21, 196. 
80. Zhu, J., Sun, S., and Zhou, X. (2021). SPARK-X: non-parametric modeling enables scalable and robust detection of spatial expression patterns for large spatial transcriptomic studies. Genome Biol 22, 184.

81. Jiaqiang Zhu, S.S., and Xiang Zhou (2021). Non-parametric modeling enables scalable and robust detection of spatial expression patterns for large spatial transcriptomic studies. . biorxiv.

82. Wolf, F.A., Angerer, P., and Theis, F.J. (2018). SCANPY: large-scale single-cell gene expression data analysis. Genome Biol 19, 15.

83. Zheng, G.X., Terry, J.M., Belgrader, P., Ryvkin, P., Bent, Z.W., Wilson, R., Ziraldo, S.B., Wheeler, T.D., McDermott, G.P., Zhu, J., et al. (2017). Massively parallel digital transcriptional profiling of single cells. Nat Commun 8, 14049.

84. Ding, J., Condon, A., and Shah, S.P. (2018). Interpretable dimensionality reduction of single cell transcriptome data with deep generative models. Nat Commun 9, 2002.

85. Lin, C., Jain, S., Kim, H., and Bar-Joseph, Z. (2017). Using neural networks for reducing the dimensions of single-cell RNA-Seq data. Nucleic Acids Research 45.

86. Lopez, R., Regier, J., Cole, M.B., Jordan, M.I., and Yosef, N. (2018). Deep generative modeling for single-cell transcriptomics. Nat Methods 15, 1053-1058.

87. Eraslan, G., Simon, L.M., Mircea, M., Mueller, N.S., and Theis, F.J. (2019). Single-cell RNA-seq denoising using a deep count autoencoder. Nat Commun 10.

88. Demsar, U., Harris, P., Brunsdon, C., Fotheringham, A.S., and McLoone, S. (2013). Principal Component Analysis on Spatial Data: An Overview. Ann Assoc Am Geogr 103, 106-128.

89. Harris, P., Brunsdon, C., and Charlton, M. (2011). Geographically weighted principal components analysis. Int J Geogr Inf Sci 25, 1717-1736.

90. Jombart, T., Devillard, S., Dufour, A.B., and Pontier, D. (2008). Revealing cryptic spatial patterns in genetic variability by a new multivariate method. Heredity (Edinb) 101, 92103.

91. Butler, A., Hoffman, P., Smibert, P., Papalexi, E., and Satija, R. (2018). Integrating single-cell transcriptomic data across different conditions, technologies, and species. Nat Biotechnol 36, 411-420.

92. Hafemeister, C., and Satija, R. (2019). Normalization and variance stabilization of single-cell RNA-seq data using regularized negative binomial regression. Genome Biology 20.

93. Tipping, M.E., and Bishop, C.M. (1999). Probabilistic principal component analysis. J Roy Stat Soc B 61, 611-622.

94. Berlin, K.S., Parra, G.R., and Williams, N.A. (2014). An introduction to latent variable mixture modeling (part 2): longitudinal latent class growth analysis and growth mixture models. J Pediatr Psychol 39, 188-203.

95. Comon, P. (1994). Independent Component Analysis, a New Concept. Signal Process 36, 287-314.

96. Gu, M.Y., and Shen, W.N. (2020). Generalized probabilistic principal component analysis of correlated data. J Mach Learn Res 21.

97. van der Vaart, A.W., and van Zanten, J.H. (2009). Adaptive Bayesian Estimation Using a Gaussian Random Field with Inverse Gamma Bandwidth. Ann Stat 37, 2655-2675.

98. Bhattacharya, A., and Pati, D. (2017). Posterior contraction in Gaussian process regression using Wasserstein approximations. Inf Inference 6, 416-440. 
1485

1486

1487

1488

1489

1490

1491

1492

1493

1494

1495

1496

1497

1498

1499

1500

1501

1502

1503

1504

1505

1506

1507

1508

1509

1510

1511

1512

1513

1514

1515

1516

1517

1518

1519

1520

1521

1522

1523

1524

1525

1526

1527

99. Silverman, B.W. (1986). Density estimation for statistics and data analysis.(London ; New York: Chapman and Hall).

100. Sheather, S.J., and Jones, M.C. (1991). A Reliable Data-Based Bandwidth Selection Method for Kernel Density-Estimation. J Roy Stat Soc B Met 53, 683-690.

101. Zhu, Q., Shah, S., Dries, R., Cai, L., and Yuan, G.C. (2018). Identification of spatially associated subpopulations by combining scRNAseq and sequential fluorescence in situ hybridization data. Nat Biotechnol.

102. Zhong, S., Zhang, S., Fan, X., Wu, Q., Yan, L., Dong, J., Zhang, H., Li, L., Sun, L., Pan, N., et al. (2018). A single-cell RNA-seq survey of the developmental landscape of the human prefrontal cortex. Nature 555, 524-528.

103. Zappia, L., Phipson, B., and Oshlack, A. (2017). Splatter: simulation of single-cell RNA sequencing data. Genome Biol 18, 174.

104. Zhao, E., Stone, M.R., Ren, X., Guenthoer, J., Smythe, K.S., Pulliam, T., Williams, S.R., Uytingco, C.R., Taylor, S.E.B., Nghiem, P., et al. (2021). Spatial transcriptomics at subspot resolution with BayesSpace. Nat Biotechnol 39, 1375-1384.

105. Lein, E.S., Hawrylycz, M.J., Ao, N., Ayres, M., Bensinger, A., Bernard, A., Boe, A.F., Boguski, M.S., Brockway, K.S., Byrnes, E.J., et al. (2007). Genome-wide atlas of gene expression in the adult mouse brain. Nature 445, 168-176.

106. Sudarov, A., Turnbull, R.K., Kim, E.J., Lebel-Potter, M., Guillemot, F., and Joyner, A.L. (2011). Ascl1 genetics reveals insights into cerebellum local circuit assembly. Dev Biol 356, 238-238.

107. Schilling, K., Oberdick, J., Rossi, F., and Baader, S.L. (2008). Besides Purkinje cells and granule neurons: an appraisal of the cell biology of the interneurons of the cerebellar cortex. Histochem Cell Biol 130, 601-615.

108. White, J.J., and Sillitoe, R.V. (2013). Development of the cerebellum: from gene expression patterns to circuit maps. Wires Dev Biol 2, 149-164.

109. Shen, Y. (2019). Sequencing the landscape of cerebellar gene expression. J Mol Cell Biol 11, 633-635.

110. Peng, J., Sheng, A.L., Xiao, Q., Shen, L., Ju, X.C., Zhang, M., He, S.T., Wu, C., and Luo, Z.G. (2019). Single-cell transcriptomes reveal molecular specializations of neuronal cell types in the developing cerebellum. J Mol Cell Biol 11, 636-648.

111. Butts, T., Green, M.J., and Wingate, R.J.T. (2014). Development of the cerebellum: simple steps to make a 'little brain'. Development 141, 4031-4041.

112. Rosenberg, A.B., Roco, C.M., Muscat, R.A., Kuchina, A., Sample, P., Yao, Z., Graybuck, L.T., Peeler, D.J., Mukherjee, S., Chen, W., et al. (2018). Single-cell profiling of the developing mouse brain and spinal cord with split-pool barcoding. Science 360, 176-182.

113. Schroder, H., Moser, N., Huggenberger, S., and SpringerLink (Online service). Neuroanatomy of the mouse : an introduction.

114. Zeisel, A., Hochgerner, H., Lonnerberg, P., Johnsson, A., Memic, F., van der Zwan, J., Haring, M., Braun, E., Borm, L.E., La Manno, G., et al. (2018). Molecular Architecture of the Mouse Nervous System. Cell 174, 999-1014 e1022.

115. Yoshikawa, F., Sato, Y., Tohyama, K., Akagi, T., Hashikawa, T., Nagakura-Takagi, Y., Sekine, Y., Morita, N., Baba, H., Suzuki, Y., et al. (2008). Opalin, a transmembrane 
sialylglycoprotein located in the central nervous system myelin paranodal loop membrane. J Biol Chem 283, 20830-20840.

116. Tekko, T., Lakspere, T., Allikalt, A., End, J., Kolvart, K.R., Jagomae, T., Terasmaa, A., Philips, M.A., Visnapuu, T., Vaartnou, F., et al. (2017). Wfs1 is expressed in dopaminoceptive regions of the amniote brain and modulates levels of D1-like receptors. Plos One 12, e0172825.

117. Matsuda, K., Budisantoso, T., Mitakidis, N., Sugaya, Y., Miura, E., Kakegawa, W., Yamasaki, M., Konno, K., Uchigashima, M., Abe, M., et al. (2016). Transsynaptic Modulation of Kainate Receptor Functions by C1q-like Proteins. Neuron 90, 752-767.

118. Salmén, F., Vickovic, S., Larsson, L., Stenbeck, L., Vallon-Christersson, J., Ehinger, A., Häkkinen, J., Borg, Å., Frisén, J., and Ståhl, P.L. (2018). Multidimensional transcriptomics provides detailed information about immune cell distribution and identity in HER2+ breast tumors. BioRxiv, 358937.

119. Sun, H., Zhou, X., Wang, X., Xu, L., Zhang, J., Jiang, K., Shang, L., Hu, Z., and Chu, J. (2020). $\mathrm{P}-\mathrm{N}$ conversion of charge carrier types and high photoresponsive performance of composition modulated ternary alloy W(SxSe1-x)2 field-effect transistors. Nanoscale 12, $15304-15317$.

120. Gerbrands, J.J. (1981). On the Relationships between Svd, Klt and Pca. Pattern Recogn 14, 375-381.

121. Lin, X., and Boutros, P.C. (2020). Optimization and expansion of non-negative matrix factorization. BMC Bioinformatics 21, 7.

122. Ertoz, L., Michael Steinbach, and Vipin Kumar. (2002). A new shared nearest neighbor clustering algorithm and its applications. In Workshop on clustering high dimensional data and its applications at 2nd SIAM international conference on data mining, pp. 105115.

123. Lun, A.T., McCarthy, D.J., and Marioni, J.C. (2016). A step-by-step workflow for low-level analysis of single-cell RNA-seq data with Bioconductor. F1000Res 5, 2122.

124. Aaron Lun, S.H. (2021). bluster: Clustering Algorithms for Bioconductor. Bioconductor.

125. Li, S.a.L., Maintainer Shengqiao. (2012). Package 'FNN'. entropy 17.

126. Nepusz, G.C.a.T. (2006). The igraph software package for complex network research. InterJournal Complex Systems, 1695.

127. Blashfield, R.K. (1991). Finding Groups in Data - an Introduction to Cluster-Analysis Kaufman,L, Rousseeuw,Pj. J Classif 8, 277-279.

128. Hubert, L., and Arabie, P. (1985). Comparing Partitions. J Classif 2, 193-218.

129. Strehl, A., and Joydeep Ghosh. (2002). Cluster ensembles --- a knowledge reuse framework for combining multiple partitions. J Mach Learn Res 3.Dec 583-617.

130. McFadden, D., and University of California Berkeley. Institute of Urban \& Regional Development. (1973). Conditional logit analysis of qualitative choice behavior.(Berkeley, Calif.: Institute of Urban and Regional Development, University of California).

131. Korsunsky, I., Millard, N., Fan, J., Slowikowski, K., Zhang, F., Wei, K., Baglaenko, Y., Brenner, M., Loh, P.R., and Raychaudhuri, S. (2019). Fast, sensitive and accurate integration of single-cell data with Harmony. Nat Methods 16, 1289-1296. 
132. Guo, L., Hu, Z., Zhao, C., Xu, X., Wang, S., Xu, J., Dong, J., and Cai, Z. (2021). Data Filtering and Its Prioritization in Pipelines for Spatial Segmentation of Mass Spectrometry Imaging. Anal Chem 93, 4788-4793. molecules in imaging mass spectrometry. Bioinformatics 29, 2335-2342.

134. Cable, D.M., Murray, E., Zou, L.S., Goeva, A., Macosko, E.Z., Chen, F., and Irizarry, R.A. (2021). Robust decomposition of cell type mixtures in spatial transcriptomics. Nat Biotechnol.

135. Saunders, A., Macosko, E.Z., Wysoker, A., Goldman, M., Krienen, F.M., de Rivera, H., Bien, E., Baum, M., Bortolin, L., Wang, S.Y., et al. (2018). Molecular Diversity and Specializations among the Cells of the Adult Mouse Brain. Cell 174, 1015-+.

136. Azizi, E., Carr, A.J., Plitas, G., Cornish, A.E., Konopacki, C., Prabhakaran, S., Nainys, J., Wu, K., Kiseliovas, V., Setty, M., et al. (2018). Single-Cell Map of Diverse Immune Phenotypes in the Breast Tumor Microenvironment. Cell 174, 1293-1308 e1236.

137. Finak, G., McDavid, A., Yajima, M., Deng, J., Gersuk, V., Shalek, A.K., Slichter, C.K., Miller, H.W., McElrath, M.J., Prlic, M., et al. (2015). MAST: a flexible statistical framework for assessing transcriptional changes and characterizing heterogeneity in single-cell RNA sequencing data. Genome Biol 16, 278.

138. Raudvere, U., Kolberg, L., Kuzmin, I., Arak, T., Adler, P., Peterson, H., and Vilo, J. (2019). g:Profiler: a web server for functional enrichment analysis and conversions of gene lists (2019 update). Nucleic Acids Res 47, W191-W198.

139. Liberzon, A. (2014). A description of the Molecular Signatures Database (MSigDB) Web site. Methods Mol Biol 1150, 153-160.

140. Liberzon, A., Birger, C., Thorvaldsdottir, H., Ghandi, M., Mesirov, J.P., and Tamayo, P. (2015). The Molecular Signatures Database (MSigDB) hallmark gene set collection. Cell Syst 1, 417-425.

141. Bult, C.J., Blake, J.A., Smith, C.L., Kadin, J.A., Richardson, J.E., and Mouse Genome Database, G. (2019). Mouse Genome Database (MGD) 2019. Nucleic Acids Res 47, D801D806. 